\title{
Applications of deep-learning approaches in horticultural research: a review
}

\author{
Biyun Yang (1) and Yong $\mathrm{Xu}^{1,2}$
}

\begin{abstract}
Deep learning is known as a promising multifunctional tool for processing images and other big data. By assimilating large amounts of heterogeneous data, deep-learning technology provides reliable prediction results for complex and uncertain phenomena. Recently, it has been increasingly used by horticultural researchers to make sense of the large datasets produced during planting and postharvest processes. In this paper, we provided a brief introduction to deeplearning approaches and reviewed 71 recent research works in which deep-learning technologies were applied in the horticultural domain for variety recognition, yield estimation, quality detection, stress phenotyping detection, growth monitoring, and other tasks. We described in detail the application scenarios reported in the relevant literature, along with the applied models and frameworks, the used data, and the overall performance results. Finally, we discussed the current challenges and future trends of deep learning in horticultural research. The aim of this review is to assist researchers and provide guidance for them to fully understand the strengths and possible weaknesses when applying deep learning in horticultural sectors. We also hope that this review will encourage researchers to explore some significant examples of deep learning in horticultural science and will promote the advancement of intelligent horticulture.
\end{abstract}

\section{Introduction}

Horticultural crops are an important part of our daily life and mainly include fruits, vegetables, materials for beverages and fragrances, herbal medicine, and ornamental plants. With the progress of modern society, horticultural crops not only play an economic role in providing foods but also play a social role in shaping human culture, beautifying landscapes, and influencing the lifestyles of humans ${ }^{1,2}$. This change in roles, which is becoming increasingly important, has driven horticultural workers to produce more varieties and better products. It also encourages horticultural researchers to do more practical work to improve the functional applications of horticultural crops.

\footnotetext{
Correspondence: Yong Xu (y.xu@fafu.edu.cn)

${ }^{1}$ College of Mechanical and Electronic Engineering, Fujian Agriculture and Forestry University, 350002 Fuzhou, China

${ }^{2}$ Institute of Machine Learning and Intelligent Science, Fujian University of Technology, 33 Xuefu South Road, 350118 Fuzhou, China
}

However, in the process of planting horticultural crops, much delicate work needs to be done manually and relies heavily on experienced workers completing jobs, such as pruning branches, thinning flowers and fruit, picking fruit, and preventing insect and pest infestations. Unfortunately, many young people are no longer engaged in gardening; however, with the progress of technology, many advanced and automatic instruments and equipment have been developed and applied to horticulture. To meet the forthcoming demands and challenges, horticultural researchers need to divert their attention towards new technologies to help make better orchard management decisions and revolutionize horticultural productivity. Therefore, producing high-quality fruits, vegetables and ornamental crops by employing advanced technologies, equipment and systems to reduce the use of human force and to improve its efficiency is the primary goal of intelligent horticulture. The rapid, accurate and automatic identification of horticultural crops and the acquisition of planting and postharvest data are important

\section{(c) The Author(s) 2021}

(c) (i) Open Access This article is licensed under a Creative Commons Attribution 4.0 International License, which permits use, sharing, adaptation, distribution and reproduction cc) in any medium or format, as long as you give appropriate credit to the original author(s) and the source, provide a link to the Creative Commons license, and indicate if changes were made. The images or other third party material in this article are included in the article's Creative Commons license, unless indicated otherwise in a credit line to the material. If material is not included in the article's Creative Commons license and your intended use is not permitted by statutory regulation or exceeds the permitted use, you will need to obtain permission directly from the copyright holder. To view a copy of this license, visit http://creativecommons.org/licenses/by/4.0/. 
directions of intelligent horticulture ${ }^{3,4}$. Based on new computer technologies and data analysis methods, the development of intelligent systems has provided golden opportunities to improve the cultivation and management of horticulture crops ${ }^{4}$.

The collection of information from orchards or groves has been increasingly facilitated in the past few decades owing to the advancement of various types of sensors in the range of digital horticulture ${ }^{3}$. Modern techniques, including remote sensing, which is implemented by means of unmanned aerial vehicles (UAVs), planes and satellites; the Internet of Things (IoT); thermal and nearinfrared cameras; and X-ray imaging technology, have been widely used to collect different kinds of digital information from horticultural $\mathrm{crops}^{3,5}$. Based on the collected data, researchers have built models and have applied them to actual production processes. For example, when the growth of horticultural crops is monitored, the growth status of crops can be judged by the established model so that optimal management decisions can be made to optimize the growth process.

However, with the rapid increase in the capability, miniaturization, and sophistication of imaging sensors, a large amount of digital horticultural information has been collected $^{3,5,6}$; therefore, the horticultural science community is facing inundation by a large amount of data, and the data themselves contain various irrelevant and redundant information ${ }^{6,7}$. Thus, creating suitable analytical technologies for such data is extremely important, and the need to deal with and to extract useful features from such uncleaned data is urgent. It is also a practical challenge to convert these technologies into real-world applications.

To date, different kinds of data analysis approaches, including machine-learning approaches, such as partial least squares $(\mathrm{PLS})^{8}$, artificial neural networks $(\mathrm{ANNs})^{9}$, support vector machines $(\mathrm{SVMs})^{10}$, random forests $(\mathrm{RFs})^{11}$, and $\mathrm{k}$-nearest neighbors $(\mathrm{KNNs})^{1,12-14}$, have been developed to tackle the challenges caused by the large amount of heterogeneous data. These approaches have shown great value in processing big data. As a subset of the machine-learning approaches, deep learning has also been widely employed, and has attracted more attention from various domains, such as agricultural production ${ }^{15-17}$, food science ${ }^{7,18}$, robotics ${ }^{19,20}$, and human action and speech recognition ${ }^{21,22}$. As an emerging versatile tool for assimilating large amounts of heterogeneous data, deep learning is able to provide reliable prediction results for complex and uncertain phenomena ${ }^{6}$. In contrast to traditional machine-learning approaches, deep learning contains "deeper" layers in its network structures that provide hierarchical representations for the data by means of various convolutions ${ }^{16,23}$. Deep-learning approaches have shown significant advantages in processing different kinds of big data collected by digital cameras and spectroscopy and have achieved better performance and higher precision than other machine-learning approaches.

Presently, deep learning has already been introduced to horticultural sectors to analyze RGB and spectral images collected from horticultural crops ${ }^{9,11}$. The authors were encouraged to prepare this survey because deep learning has been applied in horticultural science with promising results in $\sim 70$ studies. Moreover, since understanding the principles and practical applications of deep learning is not an easy task for researchers and workers in horticultural sectors, many studies are still in development. The aim of this survey is to comprehensively present an overview of the most recent research advances in the application of deep learning to horticultural sciences and provide overall guidance for researchers in this field.

\section{Brief overview of deep learning}

Machine learning is a promising tool for data processing $^{24}$. However, traditional machine-learning methods often require manual feature extraction. With the increase in the amount of large datasets and the advent of graphics processing units (GPUs), algorithmic techniques and methods have been steadily improved. Deep learning was extended from classical machine learning by adding some "deeper" (more complex) structures to models to automatically achieve feature extraction from raw data and has shown better performance than traditional machine learning for some classification and prediction problems $^{24}$. By applying different levels of abstraction layers, various nonlinear functions can be applied to allow data to be represented in a hierarchical way ${ }^{16,25,26}$. This characteristic has proven useful in improving the modeling performance for many large-scale data analysis tasks ${ }^{27}$.

Deep learning is essentially a kind of nonlinear information processing technique based on representation learning and pattern analysis. Typically, deep-learning models are built to refine multilevel representations with multilayer neural networks. Neural networks are generally composed of multiple neurons arranged in layers. Two adjacent layers are connected by neurons in terms of weights that need to be trained to learn maps, which are usually complex, from inputs, which are generally preextracted and processed data or features, to outputs or labels. Neurons that essentially represent various nonlinear functions and transformations are used to build complex models. By connecting more layers of neurons to form more complicated models that allow massive parallel connections, deep learning can solve complex real-world problems in a rapid and effective way ${ }^{16,28}$.

The property of a highly hierarchical structure along with the massive learning capability of deep-learning models enables them to carry out predictions and classifications particularly well with good flexibility and 
adaptability to a wide range of highly complicated data analysis tasks ${ }^{28}$. With the robust capability of automatic feature learning, many complex problems in the field of horticultural science can be solved in an effective and rapid way by utilizing deep-learning methods, including various recognition ${ }^{29-31}$, yield estimation ${ }^{32,33}$, quality detection $^{27,34}$, stress phenotyping detection ${ }^{35,36}$, growth monitoring $^{37,38}$, and other applications ${ }^{39,40}$. In the next section, we introduce these applications in detail.

Convolutional neural networks (CNNs) and their derived models are considered key deep-learning approaches in the field of artificial intelligence and have led to breakthroughs in image processing and analysis. $\mathrm{CNNs}$ are a family of multilayered neural networks constituting a class of deep, feed-forward artificial neural networks (ANNs) that have been successfully applied to computer vision applications ${ }^{5,25,26}$. CNNs are currently recognized as one of the most significant machinelearning approaches for big data analysis in a large variety of research $\operatorname{areas}^{28}$. Of our surveyed papers, the application of CNNs and their derivatives in horticulture accounts for a large proportion (65 papers, 92.86\%). CNNs typically contain a number of common components, including convolution, pooling and fully connected layers, in different configurations that are connected successively to perform some complex-learning tasks.

A typical deep CNN (DCNN) architecture is shown in Fig. 1. To correctly classify different species of flower images, by acquiring previous knowledge from LeCun et al. $^{24}$, Prasad et al. ${ }^{29}$ proposed a multistage CNN architecture composed of one input layer, four convolutional layers with various window sizes, five rectified linear unit (ReLU) components, two stochastic pooling layers, one densely connected layer and one layer of softmax regression output ${ }^{29}$. The input of a typical CNN is generally two-dimensional image data. The convolution layers are the core of a whole $\mathrm{CNN}$ and are composed of 2-dimensional kernels with varied weights that are moved over the image and perform feature extraction. In Fig. 1, the sizes of the four convolution kernels are set to $16^{*} 16$, $9 * 9,5 * 5$, and $5 * 5$. After convolution, a pooling layer may be used to compress the amount of information by reducing the dimensionality of the inputs to avoid overfitting. This process is achieved by substituting multiple neurons within a subsampling window with a single output neuron. In Fig. 1, 2*2 stochastic pooling is used in which every $2 * 2$ neuron in the original layer is substituted by only one random value taken from the neuron in the new layer, which reduces the number of neurons in the new layer by a factor of 4 and makes the calculation converge quickly. Finally, these features are fed to some fully connected layers for classification.

Generally, the choice of hyperparameters in training deep-learning models to a large extent determines the performance of the trained model. Important hyperparameters include the network architecture (such as the numbers of neurons in hidden layers, the structures of convolution and pooling layers and the number of layers), and contain the learning rate, weight initialization and activation function ${ }^{17}$. Although self-established

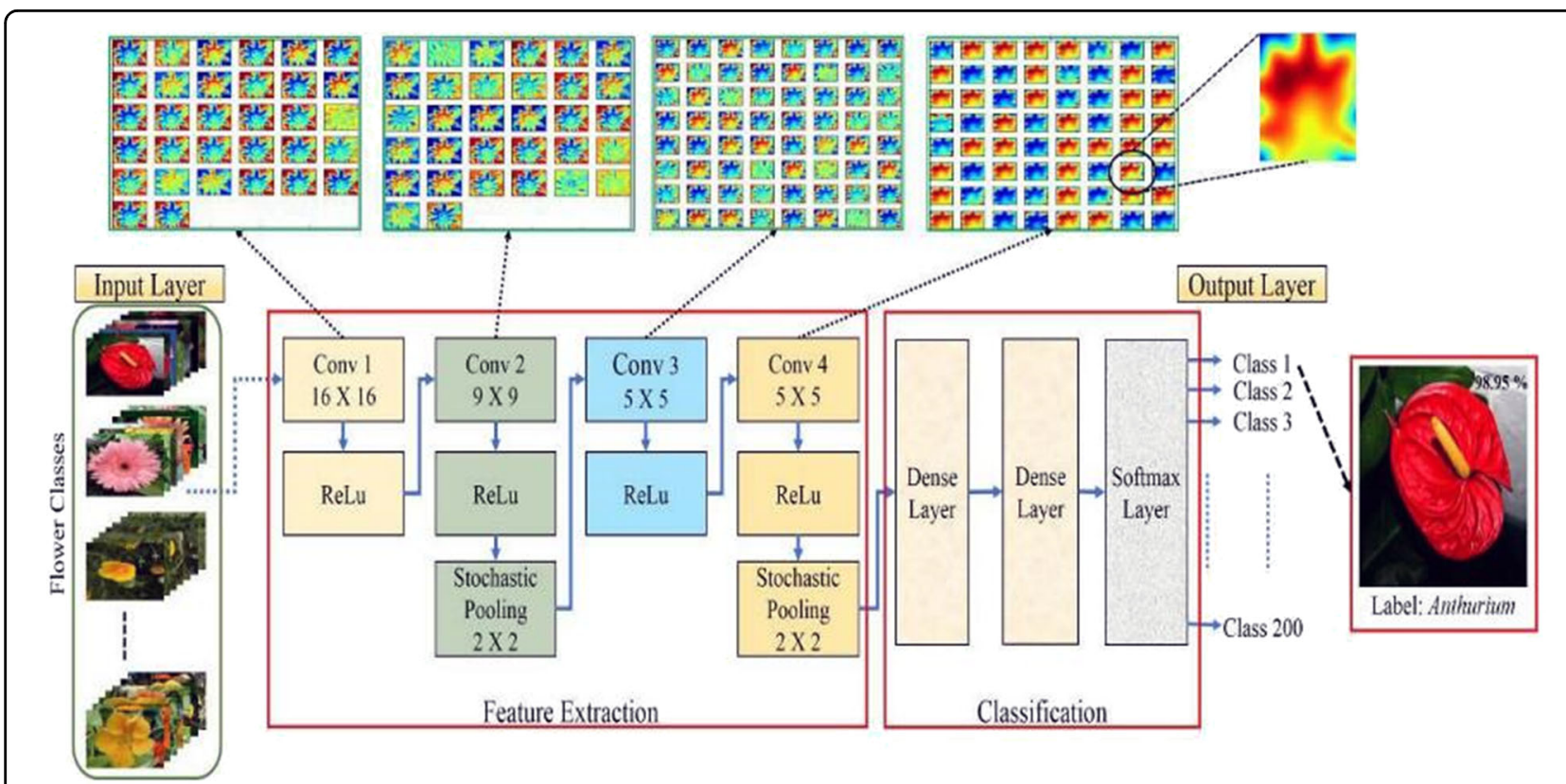

Fig. 1 A DCNN architecture. The model contains one input layer, four convolutional layers, four ReLU components, two stochastic pooling layers, two fully connected layers and one softmax regression output layer. Source: ref. ${ }^{29}$ 


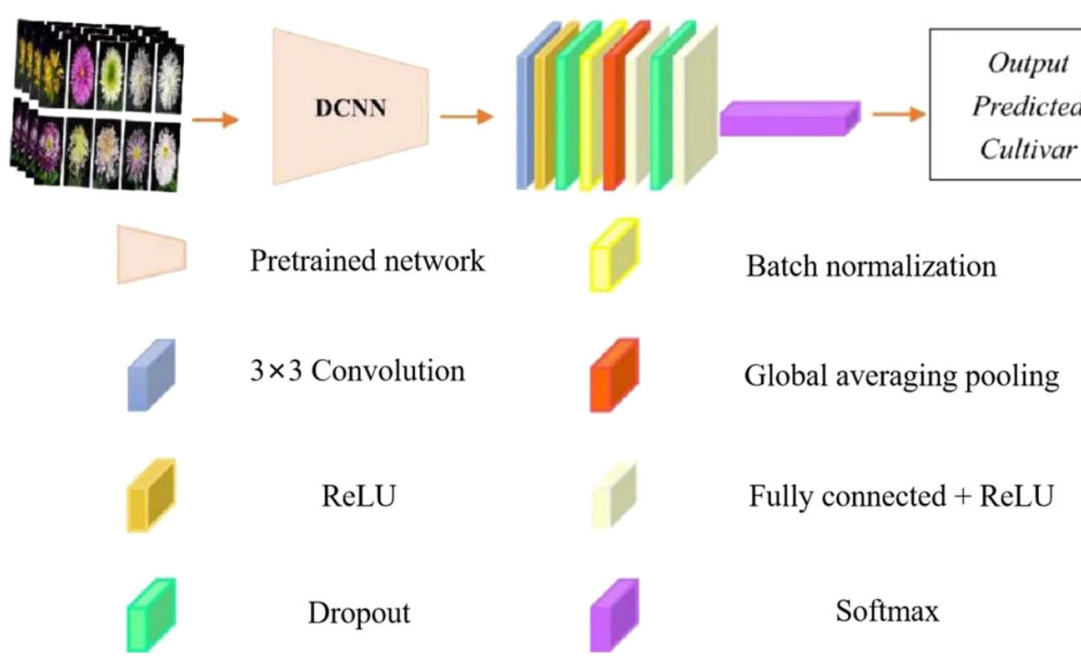

Fig. 2 A DCNN framework. The performance and stability are improved by the batch normalization layer. Overfitting is prevented by the dropout layer. Global average pooling can adapt to different input image sizes. Source: ref. ${ }^{31}$

architectures may be innovative and groundbreaking, they usually require a high level of computer literacy that is difficult for normal horticultural researchers to use. Thus, researchers often begin with a pretrained architecture that has been shown to perform well across a large variety of data structures and problems and adapt it to the problem considered, which has been demonstrated as a reliable and feasible common practice ${ }^{17,31}$. As Fig. 2 shows, feature extractors were created by the pretrained network for chrysanthemum recognition $^{31}$, the classifier consisted of two fully connected layers (each having 4096 hidden neurons), batch normalization units were used to increase DCNN performance and stability, a global averaging pooling layer was adapted to images with different input sizes, and a dropout layer was used to prevent the $\mathrm{CNN}$ model from overfitting.

Examples of $\mathrm{CNN}$ architectures that have been used for classification or regression tasks include LeNet $^{36,41}$, AlexNet $^{30,42}$, VGGNet ${ }^{43-45}$, GoogLeNet ${ }^{46}$, and ResNet $^{47,48}$ (the typical CNNs and corresponding parameters are shown in Table 1). For instance, Fig. 3 shows visual examples of flower images after each step of the Visual Geometry Group Network 16 (VGG-16) process for chrysanthemum cultivar recognition ${ }^{31}$. The network was composed of five convolutional layers, each of which was followed by one pooling layer. The convolution layers were used as filters to extract features from the input images, and the output from each convolution layer was downsampled by a pooling layer for dimensionality reduction. After the processes of five successively connected convolution and pooling layers were performed, two fully connected layers were used as a classifier to exploit the learned highly abstract features to classify the input images into predefined categories or to conduct some numerical predictions ${ }^{7,16}$. The parameters (the number of channels, activation functions, kernel size, padding strides, etc.) inside the convolution layers and the selection of the applicable models should be optimized according to the particular problem ${ }^{7}$. For example, when the VGG network was created, it was the deepest network available and showed competitive performance with only 3*3 stacked convolution kernels, which is better than using a large convolution kernel. In addition, the residual network (ResNet) incorporated local residual connections that not only improve its learning speed but also allow the network to become significantly deeper for extracting more high-level features, which enable ResNet to have a higher predictive power.

Another deep-learning model widely used for processing sequential or time-series data is the recurrent neural network $(\mathrm{RNN})^{39,49}$, which has been extensively applied in price prediction $^{49}$, natural language processing ${ }^{50}$, speech recognition $^{51}$, and other fields ${ }^{52}$. The basic principle behind an RNN is that previous information can be memorized by a network and utilized to calculate the current output. To do this, the input of the hidden layer comprises both the output from the hidden layer at the last moment, which represents the memorized previous information, and the output of the current input layer ${ }^{39}$. The tanh activation function is normally adapted in hidden neurons, while for output neurons, the activation function is generally selected according to the problem to be solved ${ }^{49}$. The feedback from the output neurons to the hidden neurons is the only loop in the RNN. The diagram of the RNN structure used for forecasting horticultural product prices is displayed in Fig. 4.

Figure 5 shows another deep-learning architecture named SAE-FNN, which is composed of a stacked autoencoder 
Yang and Xu Horticulture Research (2021)8:123

Page 5 of 31

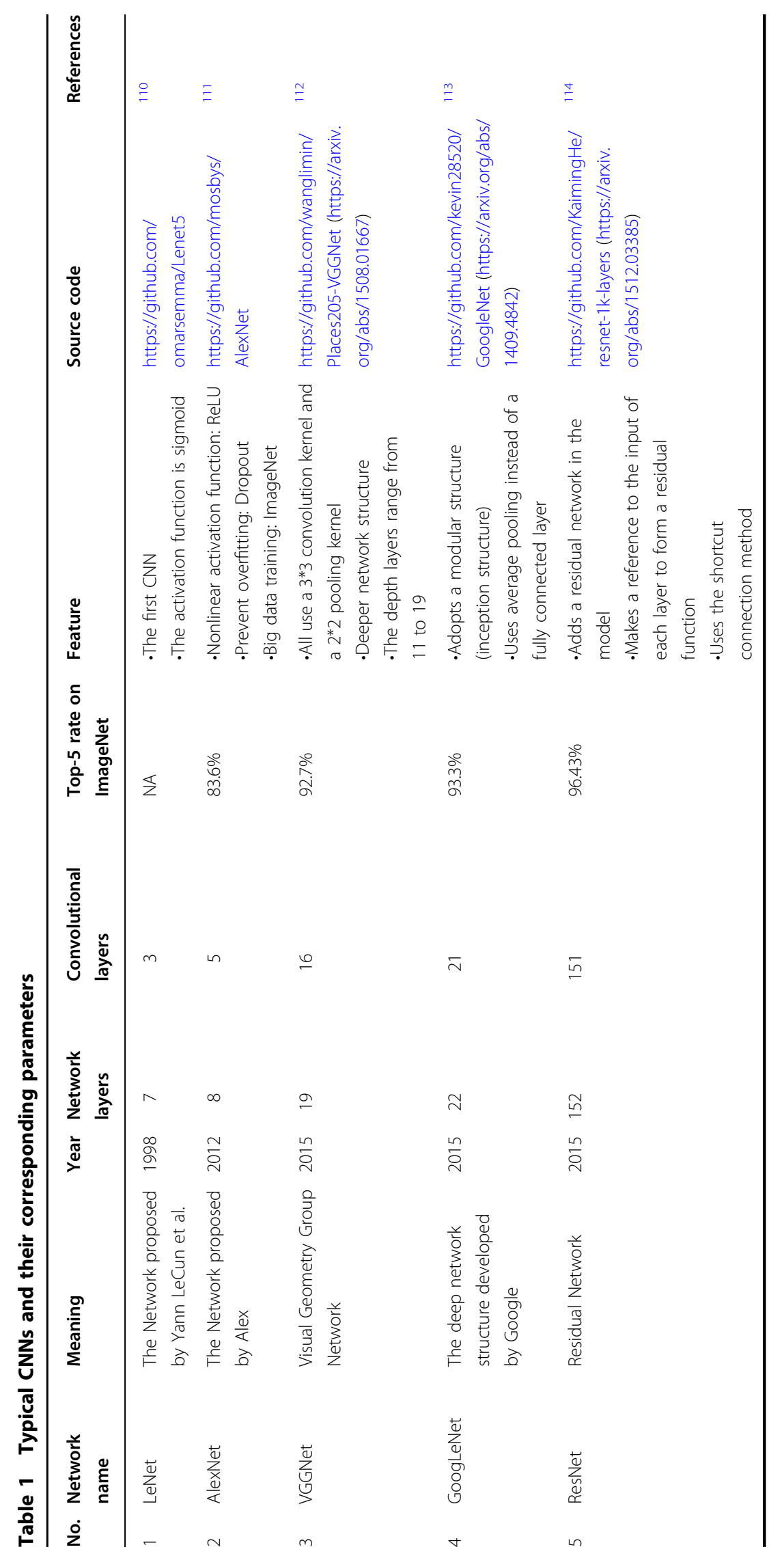




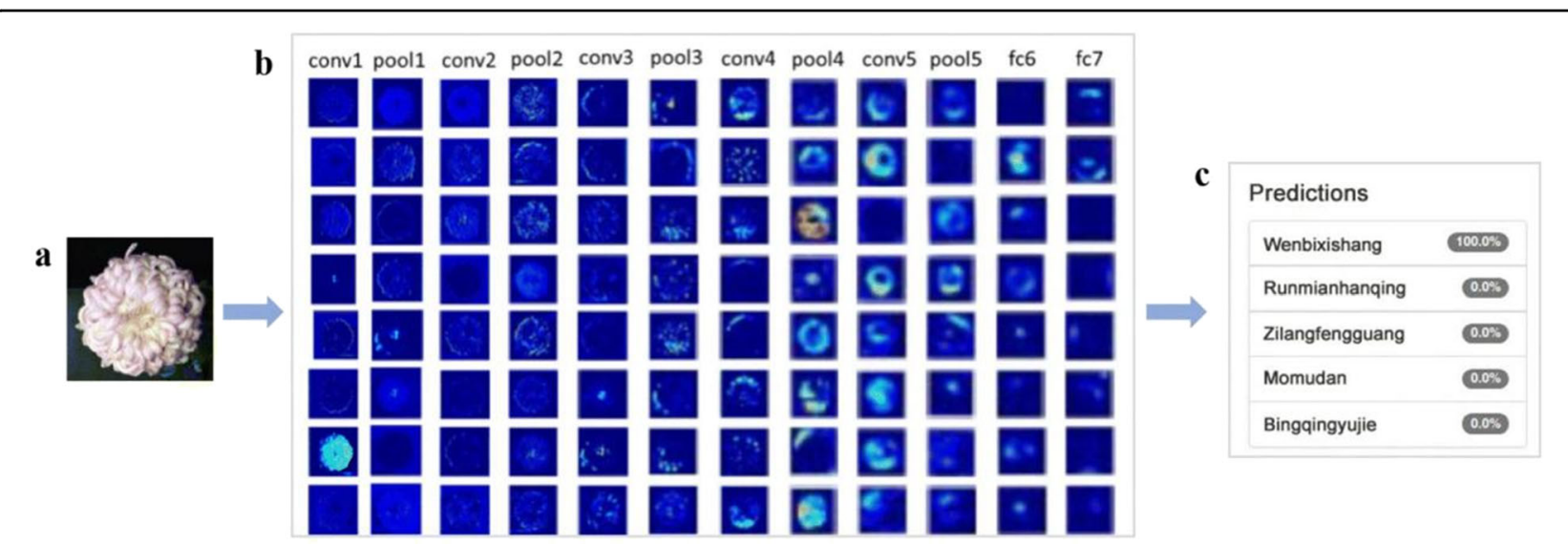

Fig. 3 VGG-16 model for image recognition. a The input images. b Visualization of the feature extraction results after each convolution (conv), pooling (pool) or fully connected (fc) layer. c The top-k prediction results. Source: ref. ${ }^{31}$

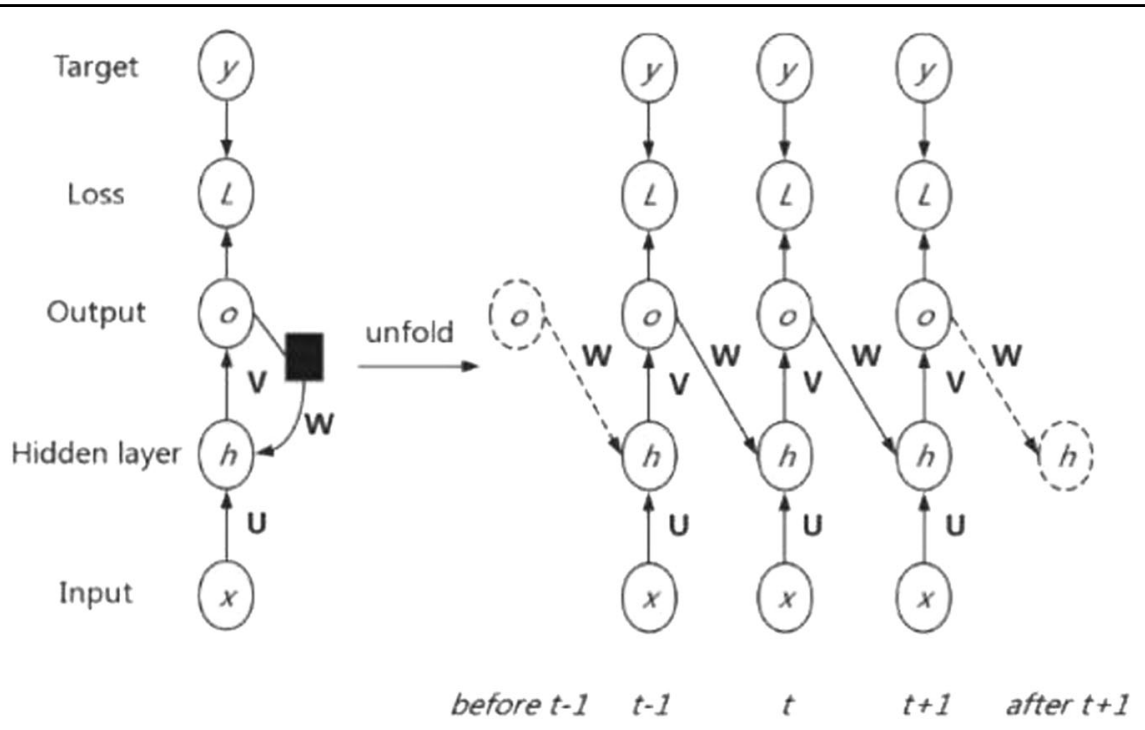

Fig. 4 Structure of an RNN. The information of the RNN propagates upwards from the initial input state. The only feedback of data is from the output neurons to the hidden neurons. The activation functions for the hidden and output neurons are the hyperbolic tangent and pure linear functions, respectively. Source: ref. ${ }^{39}$

$(\mathrm{SAE})^{53}$ and a fully connected feed-forward neural network (FNN). The network was used to predict the soluble solid content (SSC) and the firmness of a pear ${ }^{27}$. SAE uses an unsupervised approach with a basic structure consisting of an autoencoder, which is used for extracting features from the input data by the nonlinear processing of deep neural networks ${ }^{27,53}$. As Fig. 5a shows, the input was encoded into a lower dimensional vector, which was then extended again by a decoder to reconstruct the original input. Therefore, the vector, in which the decoding part is removed and the encoding part is retained, is used as the extracted feature of the input. The extracted features output from the trained network are fed to the FNN to form an SAE-FNN network for prediction tasks (shown in Fig. 5c).
In addition to being used for classification and regression tasks, deep-learning techniques have also been used for image segmentation tasks. The R-CNN method is a two-stage deep-learning object detection method (a computer vision technique for locating instances of objects in images or videos) that combines a CNN with the region proposal method. At present, the Mask R-CNN (mask region-based CNN) is the state-of-the-art R-CNN method in the field of image segmentation. By adding a branch, Mask R-CNN extends Faster R-CNN in order to exactly generate a high-quality segmentation mask on each region of interest (RoI) ${ }^{54,55}$. Figure 6 shows the architecture of the Mask R-CNN, which consists of two parts: the backbone (a feature pyramid network (FPN), 


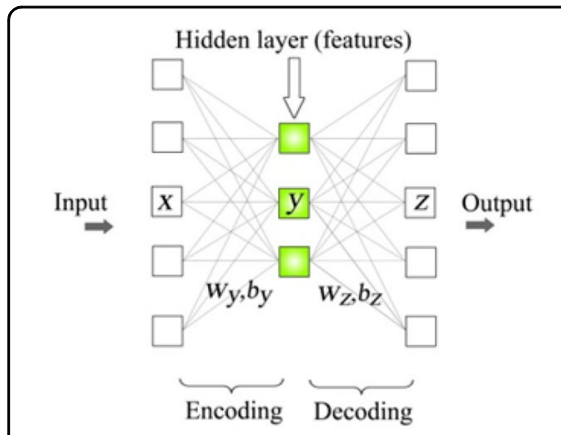

(a)

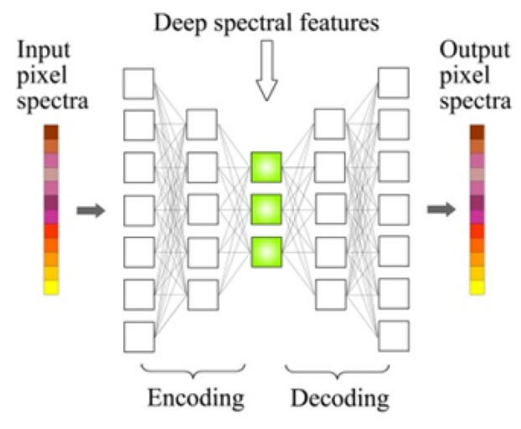

(b)

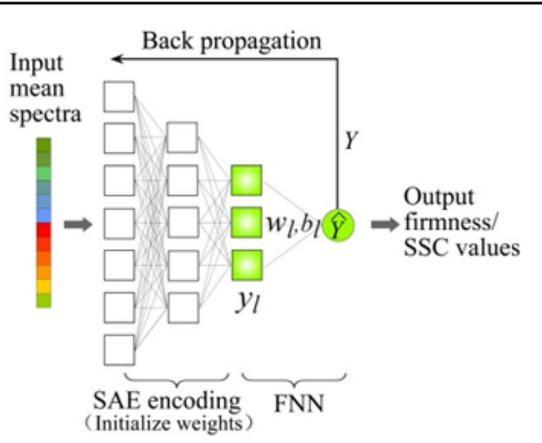

(c)

Fig. 5 The SAE-FNN architecture. a The autoencoder structure, $\mathbf{b}$ SAE is pretrained in an unsupervised manner with random pixel spectra, $\mathbf{c}$ SAEFNN is fine-tuned in a supervised manner with mean spectra and firmness (or SSC). Source: ref. ${ }^{27}$

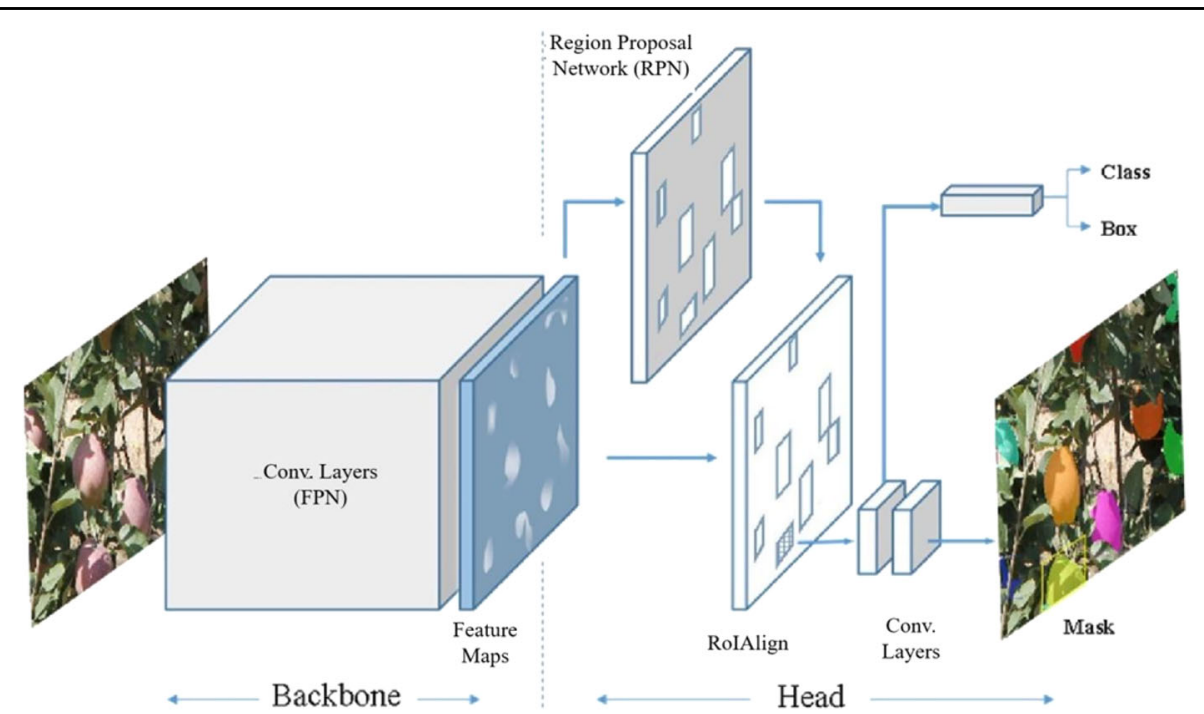

Fig. 6 The Mask R-CNN architecture. The architecture consists of two parts: the backbone and the network head. Source: ref. ${ }^{68}$

which is a fully convolutional network) for feature extraction and the network head (a small network sliding over the feature map) for bounding-box (a compact rectangular box that contains the object) recognition and mask prediction. In this figure, the Mask R-CNN model can detect and segment fruit automatically, and the mask images of the fruit are output from the model with bounding boxes. Moreover, Mask R-CNN can extract object regions from the background at the pixel level.

From the brief introduction of several commonly used models described above, we know that deep-learning technology has a powerful role in image classification, regression and segmentation. Furthermore, there are also many other kinds of network structures that are applied, such as single shot multibox detection (SSD) ${ }^{56}$, long short-term memory $(\text { LSTM) })^{32}$, you only look once (YOLO, source code is available at: http://pjreddie.com/yolo//) ${ }^{57}$, regions-CNN (R$\mathrm{CNN})^{58}$, fast region-based CNNs (Fast R-CNN, source code is available at: https://arxiv.org/abs/1504.08083) ${ }^{33}$, faster region-based CNNs (Faster-RCNN, source code is available at: https://github.com/shaoqingren/faster_rcnn (in MATLAB) and at https://github.com/rbgirshick/py-fasterrcnn (in Python) $)^{44,59,60}$, and so on. In addition, the processed data types are not simply limited to RGB images but can also include any other data forms, such as video, hyperspectral images, and spectral data.

Finally, it is helpful to introduce and describe some of the evaluation metrics used to examine network performance. Some performance metrics that were used in our reviewed papers are defined as follows:

Classification accuracy (CA): This is a measure of the number of correctly classified images/classes over the total number of images/classes for classification results. For multiclass classification problems, CA is averaged over all the classes. 
Precision: The fraction of true positives (TP) over the sum of the TP and false positives (FP). $P=\mathrm{TP} /(\mathrm{TP}+\mathrm{FP})$. Recall: The fraction of TPs over the sum of the TPs and false negatives $(\mathrm{FN}) \cdot R=\mathrm{TP} /(\mathrm{TP}+\mathrm{FN})$.

F1-score (F1): The harmonic mean (the weighted average) of precision and recall. The F1-score varies between 0 (worst) and 1 (best). F1 $=2 *(\mathrm{TP} * \mathrm{FP}) /(\mathrm{TP}+$ FP).

Root-mean square error (RMSE): Root-mean square of the differences between the predicted values and observed values.

\section{Applications of deep learning in horticulture crops}

Deep-learning technologies have been successfully applied in the horticultural domain with promising results. The application fields of deep-learning approaches in horticultural sectors include variety recognition, yield estimation, quality detection, stress phenotyping detection, growth monitoring and others. In this section of the paper, we review the specific problems addressed in the literature, the architectures and models implemented, the sources of data employed, the overall performance achieved on the basis of the metrics adopted, the comparisons with other methods, and the links to or sources for the original code for some examples. However, it is difficult to compare different studies because the datasets and performance metrics used in different studies generally vary. Thus, in this section of the paper, we focus only on comparisons of techniques applied on the same dataset within the same research paper so that the same metrics are used. Some other evaluations and comparisons among different technologies from different papers can be found in "Summary and discussions" subsection.

\section{Recognition and classification of horticultural crops}

Automatic recognition and classification of horticultural crops according to different features are the major challenges in horticultural research. The recognition of horticultural crops is a challenging task due to the great variety of crop types. There are at least 91 species of fruitbearing plants, $>200$ vegetable plants, and $>6000$ ornamental cultivars, and many cultivars are created and disappear each year ${ }^{2}$. Moreover, horticultural crops can mutate in many ways, resulting in a large amount of intraclass variation. For example, similar features can be shared within flower classes, i.e., different species of flowers can share similar shapes, colors and appearances. Therefore, classifying horticultural crops is a multiclassification problem. Even though it is feasible to conduct manual classification, it is generally very time consuming and error prone when dealing with a large number of samples ${ }^{43}$. Therefore, the application of deeplearning methods to species or cultivar recognition and classification will be an unprecedented breakthrough in horticultural research due to their high speed and robust recognition performance ${ }^{31}$.

Currently, due to the successful application of CNNs, the accuracy of image classification and event prediction has been greatly improved. With ImageNet, which is an open data resource (http://www.image-net.org/), five categories of vegetables, including broccoli, pumpkin, cauliflower, mushrooms and cucumber ${ }^{30}$, were used to train a DCNN (the AlexNet model). The experimental results showed that the accuracy rate of this DCNN model on the vegetable image dataset reached $92.1 \%$, which was a significant improvement compared with the SVM classifier $(80.5 \%)$ and the back propagation (BP) neural network (78\%) methods. Prasad et al. ${ }^{29}$ utilized 9500 images from the KLUFD and OUFD flower datasets to train a CNN model with a system architecture of four convolution layers with different filtering window sizes and employed a stochastic pooling technique for classification (see Fig. 1). The average flower recognition accuracy rate was $97.78 \%$ for the proposed $\mathrm{CNN}$ model, which is higher than those of other advanced classifiers. For flower species classification, Crbuk et al. ${ }^{61}$ employed the concatenated AlexNet and VGG-16 models to extract features, which were then used as the input to the minimum redundancy maximum relevance ( $\mathrm{mRMR}$ ) method for selecting some higher abstract features. The selected abstract features were fed into the SVM classifier that was combined with a radial base function (RBF) kernel to obtain the final classification results. They attained a 96.39\% accuracy value on Flower17, and the average accuracy was $95.70 \%$ on Flower102. Hiary et al. ${ }^{43}$ built an excellent two-step deep-learning-based model also aimed at flower type identification. The robust classifier contained two steps: an FCN (fully convolutional network) method, which was initialized by the VGG-16 model to segment the flower regions, and a $\mathrm{CNN}$ method, which was initialized by segmenting the FCN to classify the flower classes. Compared with other approaches, the proposed model achieved better learning performance and simplified the flower classification task, with classification accuracies of 98.5\%, 97.1\%, and $99.0 \%$ on Oxford 17, Oxford 102, and Zou-Nagy, respectively.

At present, several open-access image datasets with different kinds of horticultural crops, such as ImageNet and OUFD, have been collected by researchers. These large-scale image datasets are widely used and provide plenty of feature information for training deep neural network models for horticultural crop recognition. However, since horticultural crop recognition systems are still in the development stage and have not been established on a large scale, most researchers prefer to experiment with their own collected image sets. Liu et al. $^{31}$ proposed a deep-learning model with a VGG-16 network to recognize large-flowered chrysanthemums 
from two datasets collected by their own group. Dataset A comprised 14,000 images collected from 103 cultivars by an automatic image acquisition device in 2017 and was utilized to train a model and to determine the model's calibration accuracy (the top-5 rate, which is the fraction of test images for which the correct label is within the five labels considered most likely by the model, was over $98 \%$ ). Dataset B contained 197 images of the same cultivars as in dataset $\mathrm{A}$ and were obtained with a digital camera in 2008-2010 and 2016; the images were imported into the established classifier to test the model's generalization ability (the top-5 rate is above $78 \%$ ). In other applications, different models for classifying three different plum ${ }^{62}$ varieties (Angelino, BlackSplendor and Owent), six different grape ${ }^{63}$ varieties (Tinta Roriz, Tinta Amarela, Tinta Barroca, Tinto Cão, Touriga Nacional and Touriga Franca) and seven different olive ${ }^{64}$ varieties (Arbequina, Changlot Real, Arbosana, Picual, Lechín de Sevilla, Ocal and Verdial de Huévar) were proposed and used DCNNs. The experimental results showed that the proposed system achieved remarkable behavior, with an accuracy rate ranging from 91 to $97 \%$ in plum variety classification. The highest accuracy was $95.91 \%$ when applying the Inception-ResNet-V2 architecture to classify olive varieties from 2800 fruits, and the network modified from AlexNet for grape variety identification achieved a test accuracy of $77.30 \%$. In addition to using RGB images, Fernandes et al. ${ }^{65}$ used spectral information to separate the two main grapevine varieties (Touriga Franca $\left(\mathrm{TF}_{\mathrm{var}}\right.$ ) and Touriga Nacional $\left(\mathrm{TN}_{\mathrm{var}}\right)$ ). Datasets with a total number of 35,833 spectra from 64 varieties and 626 plants were collected to establish the classification model. The results showed that each model had its own merits: for $\mathrm{TN}_{\mathrm{var}}$, the SVM model achieved better experimental results than the $\mathrm{CNN}$, and $81.9 \%$ of the $\mathrm{TN}_{\mathrm{var}}$ spectra and $63.0 \%$ of the non- $\mathrm{TN}_{\mathrm{var}}$ spectra were correctly classified. For $\mathrm{TF}_{\mathrm{var}}$, the $\mathrm{CNN}$ achieved the best results, and the correct classification percentages of the $\mathrm{TF}_{\mathrm{var}}$ and the non- $\mathrm{TF}_{\mathrm{var}}$ spectra were $93.8 \%$ and $91.6 \%$, respectively.

In addition to variety identification, deep learning has also been widely used in the automatic detection of orchard fruits. The development of a reliable and accurate fruit detection system is the first step in creating an autonomous harvesting system that is a promising prospect of future agricultural applications. To develop efficient and robust fruit detection systems for orchards, many researchers have performed related studies to address some complex conditions, such as illumination variation, leaf shading, and changing appearance ${ }^{47,66}$, in working environments.

To conduct robust and efficient detection and segmentation of fruits and branches in apple orchards, Kang and his team proposed a series of neural network frameworks based on deep learning, such as LedNet (a network that utilized a FPN combined with atrous spatial pyramid pooling (ASPP) to improve the model's detection performance) with ResNet-10167 (a lightweight backbone), the DaSNet model (a network, which adopted a gated feature pyramid network (GFPN) combined with ASPP to enhance the feature extraction capability of the model) with ResNet-101 ${ }^{66}$, the DaSNet model with ResNet-50 $0^{66}$, the DaSNet-V2 model with ResNet- $101^{47}$, and the DaSNet-V2 model with ResNet- $18^{47}$ (source code: https://github.com/tensorflow/models/tree/master/ research/slim) ${ }^{66}$. The experimental results showed that LedNet with ResNet-101 achieved an accuracy of $86.4 \%$, an F1 score of 0.849 and a recall score of $84.1 \%$ for the detection of apples in orchards, and DaSNet with ResNet101 reached $77.2 \%$ and $87.6 \%$ for the semantic segmentation of branches and apples ${ }^{67}$.

Semantic segmentation in computer vision is the segmentation of different objects at the pixel level where each pixel is uniquely assigned to one object category of the image. In an instance segmentation task, all pixels associated with every object in the image should be distinguished and annotated. Instance segmentation on each fruit is necessary because it can provide abundant geometric property information (such as size and shape), and such parameters can be utilized to identify the poses of the fruits, especially for overlapped or shaded fruits. Then, they proposed an improved deep neural network called DaSNet-V ${ }^{47}$. The DaSNet-V2 model with ResNet-101 obtained a recall score of $86.8 \%$, a precision score of $88 \%$, a branch segmentation accuracy of $79.4 \%$, and an apple segmentation accuracy of $87.3 \%$. DaSNet-V2 can efficiently and robustly perform visual sensing for automatic harvesting in apple orchards. Furthermore, Gené-Mola et al. ${ }^{68}$ performed a study on apple $2 \mathrm{D}$ detection with Mask R-CNN and 3D location utilizing structure-frommotion (SfM) photogrammetry. By testing 11 normally grown Fuji apple trees comprising a total number of 1455 apples, the system achieved encouraging performance with an F1-score that increased from 0.816 for 2D detection to 0.881 for 3D detection and location.

Since fruits and vegetables have different shapes and colors, some scientists have conducted further experiments with specific horticultural products and tried to extend models to the identification and classification of other products.

Mao et al. ${ }^{69}$ proposed an automatic cucumber recognition model that combined a multipath CNN (MPCNN) with a SVM and color component selection ${ }^{69}$. The detection results showed that the truly classified rate (the rate of pixels correctly classified as true cucumber pixels) for cucumber images was above $90 \%$ and the falsely classified rate (the rate of pixels misclassified as true cucumber pixels) was $<22 \%$. Quiroz et al. ${ }^{70}$ proposed a model built on a CNN to recognize blueberry plants, and 
the detection results of the proposed model were as follows: $86 \%$ accuracy, $86 \%$ precision, $88 \%$ recall, and 0.86 F1 score. In another study, Mask R-CNN was applied to improve the detection accuracy of fruits by realizing instance segmentation and the picking point for a strawberry harvesting robot $^{54}$. The detection results from 100 test strawberry images were obtained and are as follows: 95.41\% recall, $95.78 \%$ precision, and $89.85 \%$ mean intersection over union (MIoU). For automatic localization and pose estimation, Giefer et al. $^{56}$ presented an SSD model, named Deep-6DPose (an end-to-end deeplearning framework that recovers a $6 \mathrm{D}$ object pose from a single RGB image), that was applied to objects with irregular shapes to establish an automatic fruit grading and packing system.

To acquire accurate and rapid phenotypic trait data, Csillik et al. ${ }^{71}$ and Ampatzidis et al. ${ }^{72}$ combined UAV multispectral imagery with deep-learning methods to detect citrus. The combination of a CNN workflow that utilizes the Google TensorFlow API (https://www. tensorflow.org/api_docs) with a simple linear iterative clustering (SLIC) algorithm was employed by Csillik et $\mathrm{al}^{71}$ and achieved a $96.24 \%$ accuracy; the YOLO-V3 model (source code: https://github.com/mystic123/ tensorflow-yolo-v3) was used by Ampatzidis et al. ${ }^{72}$ and achieved a $99.8 \%$ accuracy. There is another study on methods for classifying cluster crops. As a feature of horticultural products, some products are clustered and should be classified collectively rather than individually. Therefore, a noninvasive DNN classification model for clustered bananas was developed by Le et al. ${ }^{55}$ as a pioneering study based on deep learning for classifying clustered fruits. With the Mask R-CNN model, the proposed deep-learning model reached an overall classification accuracy of $96.5 \%$ with only a single side banana image as the input feature.

The techniques for horticultural crop cultivation are somewhat different from those for cultivating other common crops. Regular pruning of crops is needed, and it is also necessary to thin out flowers and fruits to maintain strong trees and to produce higher yields with better quality fruit. Segmenting branches, trunks, flowers and fruits is a key step in automating horticultural cultivation technology. Majeed et al. $^{20}$ adopted a CNN-based semantic segmentation network (SegNet) to detect branch, trunk, and trellis wire to achieve automated tree trimming in an apple orchard. In another study, Sun et al. ${ }^{73}$ used a CNN based on the original Faster R-CNN algorithm to detect and identify flowers and mature (red) and immature (green) fruits of tomatoes. $\mathrm{Hu}$ et al. ${ }^{60}$ introduced a method that combined intuitionistic fuzzy set (IFS) theory with the Faster R-CNN model to detect individual ripe tomatoes. The ripe tomato image dataset, which includes adjacent, separated, leaf-shaded, and overlapped images, was used to obtain exact values of the height and width, and these data were then analyzed to evaluate the overall performance of the proposed detection model. Based on the proposed recognition methods, the RMSE results of tomato height and width were 3.306 pixels and 2.996 pixels, respectively.

Although many researchers have conducted a large amount of research on the identification and classification of horticultural products and the proposed methods have high detection performance, modern popular deep neural networks generally require high-performance computing machines for reasoning, which is unrealistic for ordinary farms and orchards.

To reduce the computational cost of networks and meet the computational requirements of real-time devices with low-power-consumption terminal processors, Shi et al. ${ }^{74}$ proposed a generalized attribution method for pruning unnecessary connections in a channel from a welldesigned large-scale network to accurately detect mangos in a channel (the source code is available at https:// github.com/GlowingHorse/Fast-Mango-Detection). The proposed pruning method can compute the convolutional kernel attributions and fine-tune the network by retaining the important pretrained parameters to extract special mango features. Compared to the YOLO-based network without pruning, the computational cost of the proposed network was reduced by $83.4 \%$, with only an $\sim 2.4 \%$ loss in accuracy.

In the examples of the application of machine vision to horticultural crops given above, it can be seen that deeplearning methods have been applied to all aspects of horticultural research, including variety or species classification, key organ classification, and location detection. We also find that researchers' efforts to apply deeplearning technology to actual production have achieved exciting results in terms of improving performance and detection speed. Table 2 summarizes the technical details of the studies mentioned in this subsection, including the target problems, the compositions of the datasets, the preprocessing methods, the models and frameworks, and the performance comparisons with other algorithms.

\section{Yield estimate of horticultural crops}

An early and accurate estimation of the preharvest yields for horticultural products is generally required by both producers and agricultural companies to guide harvesting and marketing processes for effective and efficient decision making and forward planning. A yield estimation can actually be attributed to the object counting problem in computer vision, which has demonstrated good performance in crowd counting problems ${ }^{75}$ and other plantrelated counting problems ${ }^{76}$. In current studies, such yield estimation methods have been adopted for horticultural crops. To estimate a citrus yield, Apolo-Apolo et al. ${ }^{77}$ 


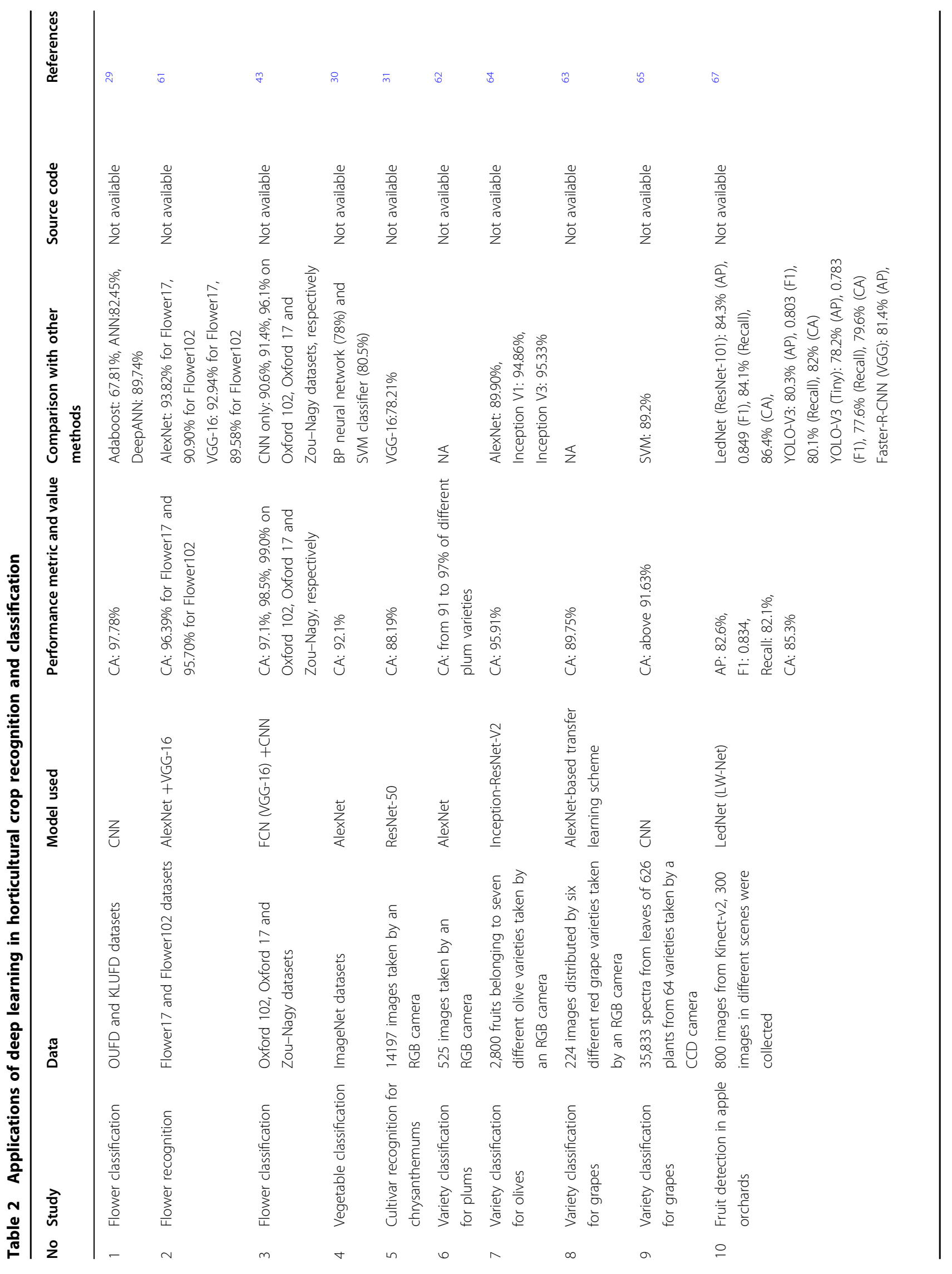




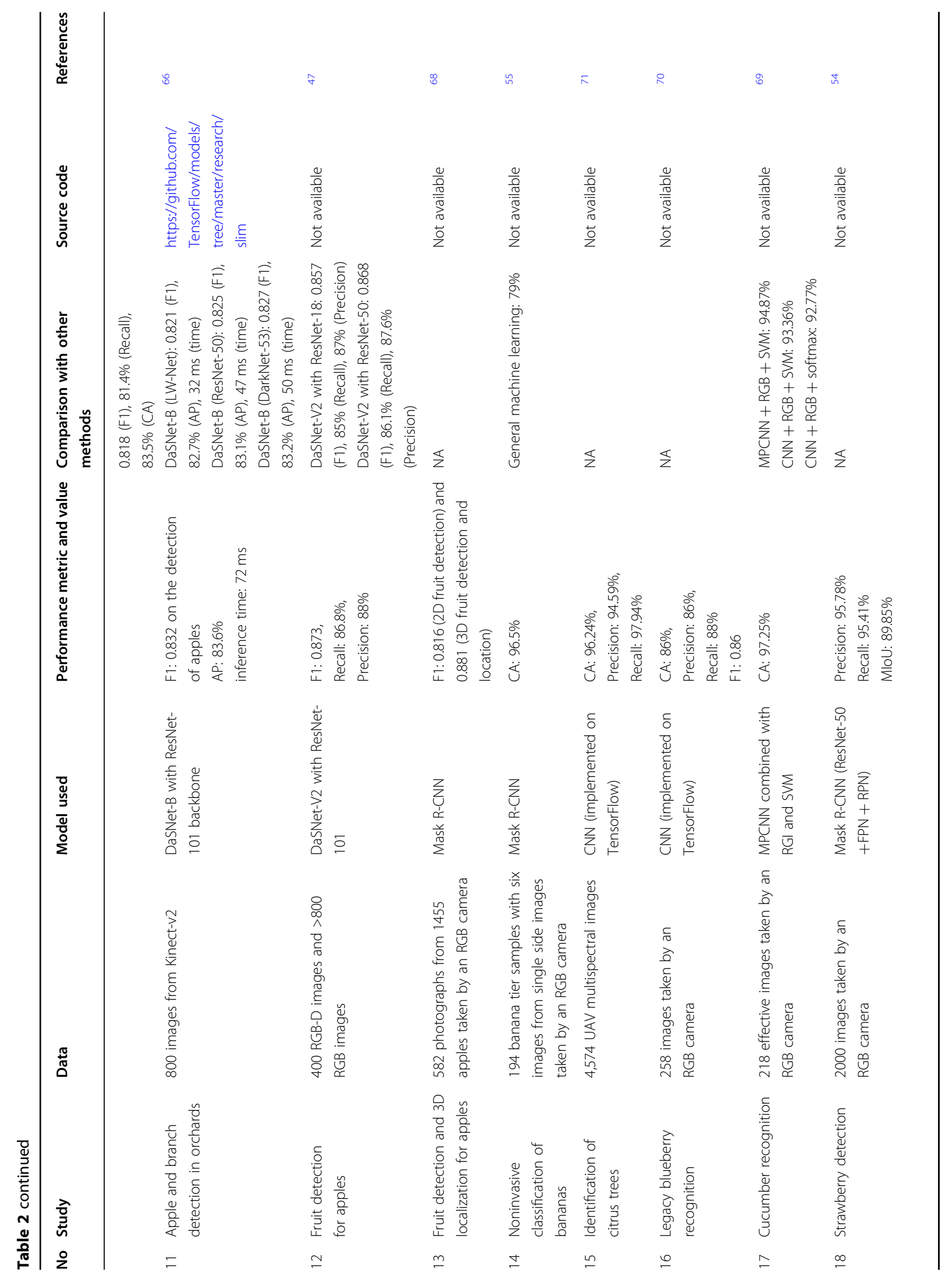


Yang and Xu Horticulture Research (2021)8:123

Page 13 of 31

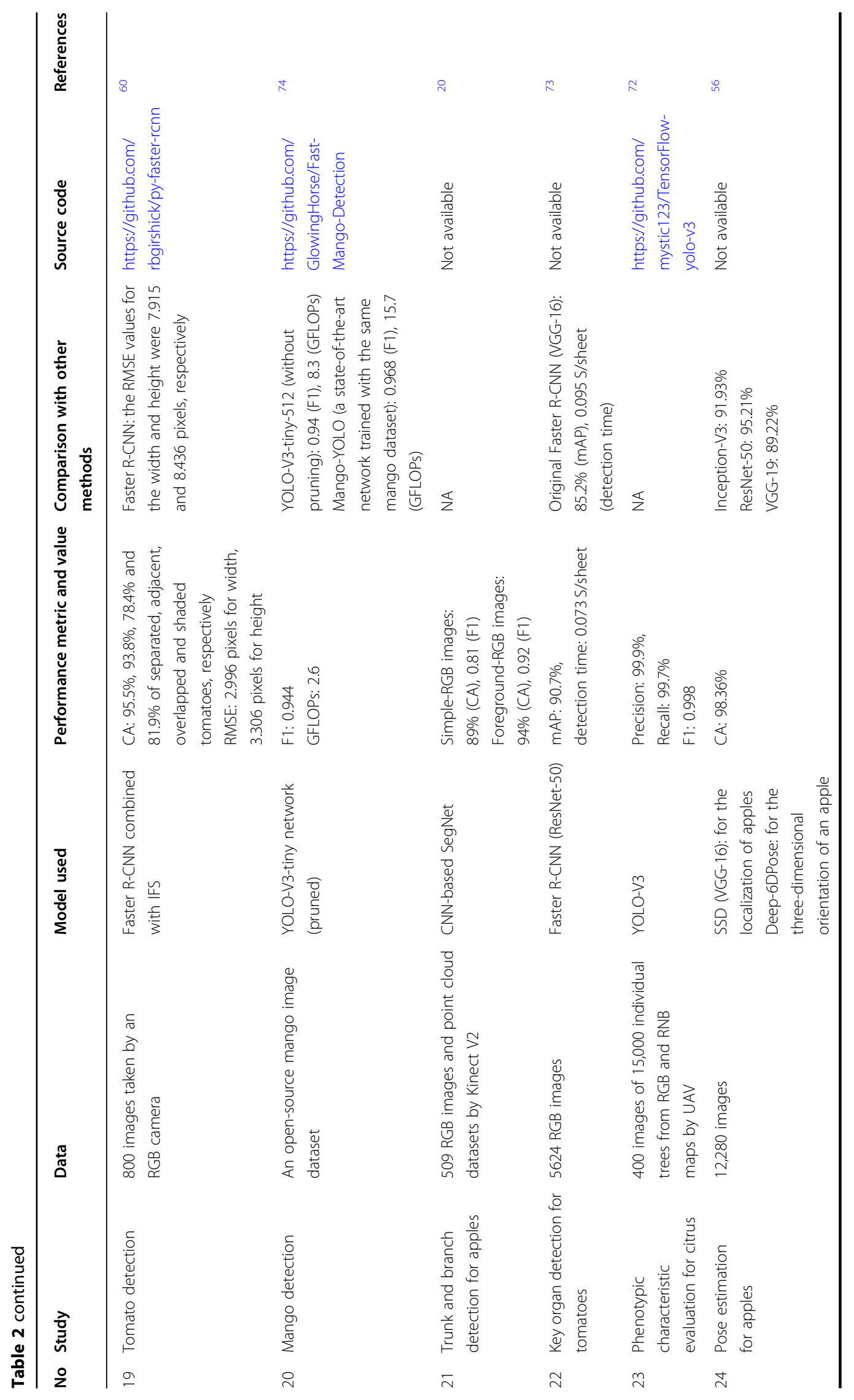


developed a methodology based on the Faster R-CNN model to detect the existence, count the number and measure the size of citrus fruits and established a network based on LSTM to estimate yields of citrus fruits. The results showed that the average standard error (SE) was only $6.59 \%$ when the manual counting result was compared with the proposed model's detection result. Furthermore, when comparing the actual yields with the estimated yields per tree, the SE was $4.53 \%$, and the standard deviation (SD) was $0.97 \mathrm{~kg}$.

Rebortera et al. ${ }^{32}$ built a deep multifaceted system that incorporated a number of LSTM layers to forecast banana harvest yields. The dataset contained 35,000 observations of banana harvest yields from approximately 2014 to 2018. The enhanced model achieved better performance, which is as follows: the RMSE was 34.805, and the error rates were decreased by $44.95 \%$ and $43.5 \%$ when compared to models that applied multiple LSTM layers and a single LSTM layer, respectively. In another study, Neupane et al. $^{78}$ developed a deep-learning algorithm (the Faster R-CNN Inception-V2 model) to accurately detect and count bananas in high-resolution RGB images acquired with a UAV ${ }^{78}$. The results showed that $75.8 \%$, $85.1 \%$ and $96.4 \%$ of bananas were correctly detected on the datasets collected from 60,50 and 40 meters above ground, respectively, on the same farm, and the recall rate reached $99 \%$ when combining the results detected from the 40- and 50-meter datasets. Moreover, as the singleview images from one side of the fruit tree would underestimate the fruit yield since fruit can be hidden by leaves or fruits, a method that applied a video tracking system was proposed and combined MangoYOLO and Kalman filters with the Hungarian algorithm for the detection, tracking, and counting of mango fruits on a tree $^{79}$. Compared to manual counting methods, the proposed video-based tracking model, which added additional imaging perspectives, detected 2050 fruits (62\% of the total harvested fruits) with a bias-corrected RMSE of 18.0 fruits per tree, while the two-sided dual-view image model (which also employed MangoYOLO) detected 1322 fruits (40\%) with a bias-corrected RMSE of 21.7 fruits per tree. Preharvest yield prediction is also very important for vegetable production. Chen et al. ${ }^{33}$ developed an automatic strawberry flower detection method for predicting yield with a small UAV equipped with an RGB camera. With this system, the mean average precision (mAP) of all detected objects was $83 \%$ at $2 \mathrm{~m}$ above the ground, and the average accuracy of counting was $84.1 \%$. Rahnemoonfar et al. ${ }^{80}$ proposed a simulated learning method for crop yield estimation by counting fruits based on synthetic tomato images. To capture features on multiple scales, they employed a modified Inception-ResNet model. The detection results showed that the average accuracy was $91 \%$ on real images and $93 \%$ on synthetic images. In another study, Afonso ${ }^{81}$ adopted the Mask R$\mathrm{CNN}$ model to detect tomatoes in a total of 123 images taken in a greenhouse; the model achieved a better performance than the classical segmentation method. The detection results of the Mask R-CNN model with a ResNeXt-101 architecture showed that the overall precision was $96 \%$, the recall score was $95 \%$ and the F1 score was 0.95 for single fruit detection, whereas when the classical segmentation method was used, these parameters were $60 \%, 80 \%$ and 0.69 , respectively.

From the above examples of applications in estimating the preharvest yields for horticultural products, we can see that researchers have obtained some good results. With the efforts of scientists, these techniques will be applied to actual production to guide planting plans, harvesting plans and marketing decisions in the future. The technical details of the studies mentioned in this subsection are summarized in Table 3.

\section{Quality detection of horticultural crops}

With improvements in living standards, people have increasingly higher expectations for the quality of daily fruits and vegetables. However, fruits and vegetables are susceptible to diseases, insect pests, mechanical damage and improper postharvest treatment during production, planting, storage, transportation, marketing, and other procedures. Both the edible and economic values of horticultural products can be reduced when damage occurs. Therefore, quality detection for horticultural products, especially for fruits and vegetables, is currently a challenging and hot field. In more recent studies, deeplearning methods combined with RGB images or spectrographic techniques have been widely employed as effective and noninvasive horticultural product quality detection approaches to tackle practical problems, including postharvest grading classification, maturity detection, bruise detection, and nutrient content prediction $^{7}$. Compared to traditional machine-learning approaches, deep learning was approaches have been applied to analyze and process image data, spectral data or sound data and have been proven to have better classification performance $^{82}$.

Nasiri et al. ${ }^{45}$ presented a method for discriminating defective dates from healthy dates based on RGB images with a VGG-16 architecture ${ }^{45}$. The introduced CNN model reached a classification accuracy of $96.98 \%$. In another study, Rosanna et al. ${ }^{83}$ explored the application of a deep-learning approach with image processing to classify banana grades and achieved above $90 \%$ accuracy. The grading classification of dates and bananas is determined primarily by visible surface defects, and the features can be expressly revealed in RGB image data. However, internal or subdermal damage and the edible quality of fruits and vegetables cannot be recognized visually ${ }^{1}$. 


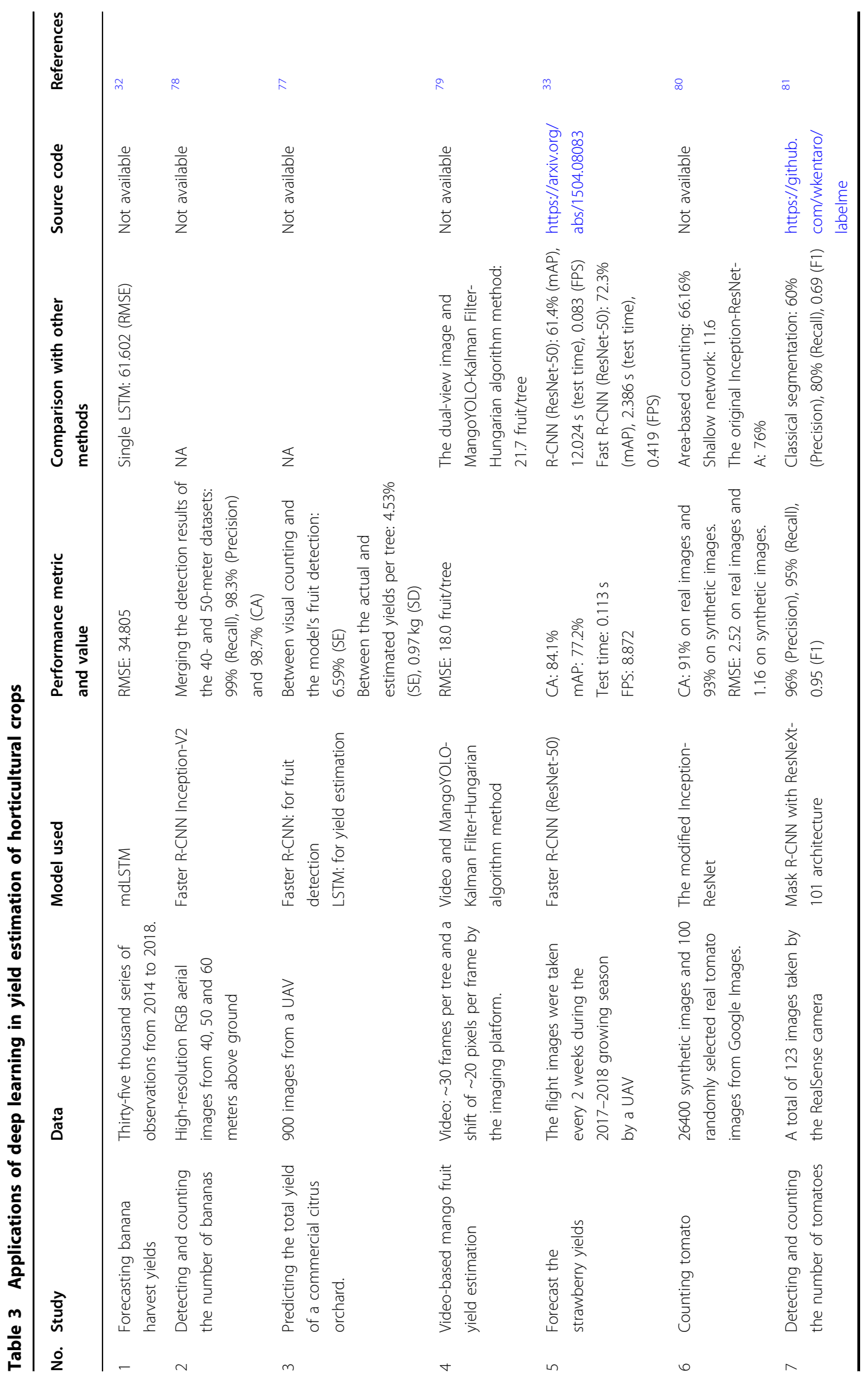


Nondestructive testing technologies, such as nearinfrared spectrographs ${ }^{84}$, hyperspectral imagers ${ }^{85,86}$ and thermal imagers ${ }^{36}$, which can detect the internal state of an object without destroying it, have been considered feasible solutions to traditional detection and analysis techniques $^{82}$. Processing large amounts of hyperspectral image data rapidly and accurately is a great challenge. Deep learning, as one of the popular machine-learning methods, has been applied to process complex, massive amounts of data. Wang et al. ${ }^{82}$ focused on the internal and invisible mechanical damage of blueberries utilizing deep-learning technology in combination with hyperspectral transmittance data. The ResNet architecture was chosen for the CNN model. Moreover, five traditional machine-learning algorithms, i.e., linear regression (LR), sequential minimal optimization (SMO), bagging and multilayer perceptron (BMP), and RF algorithms were used for comparison. The fine-tuned ResNet obtained an average accuracy of $88.0 \%$ and an F1-score of 0.90 . The deep-learning framework has great potential for detecting internal mechanical damage in fruits. In another study, Zhang et al. $^{85}$ applied the deep-learning-based FCN model to the tasks of segmentation and combined it with hyperspectral transmittance images (HSTIs) to accurately detect internal bruising in blueberries. The FCN method achieved better performance than the SVM classifier in both unbruised and bruised fruit prediction. The new fullwavelength method combined with random initialization achieved the best accuracy of $81.2 \%$ on the whole test dataset and could be utilized to investigate the resistance of blueberries to mechanical damage and other damage (the source code is available at https://github.com/UGABSAIL/BlueberryFCN.git) ${ }^{85}$. Feng et al. ${ }^{87}$ used hyperspectral imaging to detect some minor bruises on winter jujubes. LR, SVM, and CNN models were used for quantitative analyses. The CNN method obtained the highest detection performance, with most of the accuracies exceeding $85 \%$, and the prediction time was also the shortest. The overall performance results revealed the promising and meaningful potential for the detection of minor bruises on winter jujube by utilizing deep-learning methods to analyze the pixelwise NIR spectra and visible and near-infrared (Vis/NIR) spectra collected from hyperspectral imaging systems (the pseudocode of the method was given in the original paper). Liu et al. ${ }^{86}$ developed a novel classification method by applying the combination of a stacked sparse autoencoder (SSAE) with a $\mathrm{CNN}$ to detect defects in pickling cucumbers with a hyperspectral imaging-based dataset. The results showed that, compared with the SSAE model, the CNN-SSAE method improved the performance of a six-class classification task and achieved overall accuracies of $88.3 \%$ and $91.1 \%$ at conveyor speeds of 165 and $85 \mathrm{~mm} \mathrm{~s}^{-1}$, respectively.
SSC and firmness are the most significant properties of edible quality in horticultural products and directly influence consumer satisfaction ${ }^{27}$. The Vis/NIR hyperspectral imaging technique has been used for the nondestructive detection of the internal quality attributes and the chemical composition in fruits due to its high sensitivity and accuracy. The principle behind the spectral detection method is to measure the spectrum of reflected or transmitted missions from fruit and to construct a relationship between the measured spectrum and the chemical composition of the fruit. The received spectrum can then be used to indicate the SSC and/or firmness by referring to some chemometric methods ${ }^{27}$.

Bai et al. ${ }^{84}$ focused on the accurate prediction of SSC in apples collected from a number of geographical origins. A multiorigin SSC prediction method for apples was developed by the combination of NIR analysis, spectral fingerprint feature extraction, optimal wavelength selection, model search strategies, origin recognition, and multivariate regression analysis with deep learning. The correlation coefficients of prediction $\left(\mathrm{R}_{\mathrm{P}}\right)$ and RMSEs of prediction (RMSEP) values of $99.0 \%$ and $27.4 \%$, respectively, were obtained by the proposed model. In another study, Yu et al. $^{27}$ developed a deep-learning method consisting of SAE and FNN coupled with a hyperspectral imaging technique for the prediction of SSC and the firmness of postharvest pears. The proposed model obtained reasonable prediction performance with coefficients of determination of the prediction set $\left(R^{2}\right)=$ $92.1 \%$, the RMSEP $=0.22 \%$ and the ratio of the prediction to the deviation of the prediction set $\left(\mathrm{RPD}_{\mathrm{P}}\right)=3.68$ for $\mathrm{SSC}$, and $R_{\mathrm{P}}^{2}=89.0 \%$, RMSEP $=1.81 \mathrm{~N}$ and $\mathrm{RPD}_{\mathrm{P}}=3.05$ for firmness.

The laser backscattering method is another optical technique that can be used for the nondestructive detection of fruit samples. Wu et al. ${ }^{34}$ constructed an AlexNet model with an 11-layer structure, identified the defect, normal, stem and calyx regions of apples with laserinduced light backscattering imaging, and achieved a higher recognition rate of $92.5 \%$ and an accuracy better than those obtained by conventional machine-learning algorithms.

In addition to spectroscopy technology, acoustic sensing is also a reliable method for the nondestructive detection of horticultural products. Lashgari et al ${ }^{88}$ applied acoustic and deep-learning techniques to detect mealy and nonmealy apples. VGGNet and AlexNet, which are both famous pretrained $\mathrm{CNN}$ models, were used to classify the apples. The accuracies of VGGNet and AlexNet for classifying mealy and nonmealy apples were $86.94 \%$ and $91.11 \%$, respectively. Although VGGNet is deeper and performed better on ImageNet (see Table 1), in combination with an acoustic sensing system, AlexNet had a superior ability in terms of classification accuracy, training 
and classification speed compared to VGGNet in this particular work.

In commercial orchards, it is important to monitor the maturity of fruit during the whole development period to determine the optimal time to harvest. Automated machine vision techniques are widely used to detect and identify the growth and maturity stages. Wendel et al. ${ }^{8}$ proposed a novel approach that utilized a LIDAR sensor, a hyperspectral camera and a navigation system fixed to a ground vehicle for predicting the dry matter (DM) content of individual fruit from a commercial mango orchard. A cross-validation $R^{2}{ }_{\mathrm{CV}}=64 \%$ and $\mathrm{RMSE}_{\mathrm{CV}}=1.08 \% \mathrm{w} / \mathrm{w}$ were achieved by the $\mathrm{CNN}$ for fruit on trees, while a $R_{\mathrm{CV}}^{2}$ $=58 \%$ and $\mathrm{RMSE}_{\mathrm{CV}}=1.17 \% \mathrm{w} / \mathrm{w}$ were achieved by PLS. In another study, a CNN was used to evaluate citrus maturity by utilizing a fluorescent spectrum signal ${ }^{89}$. They adopted fluorescence spectroscopy to estimate the Brix/ acid ratio. As a result, the absolute error of the Brix/acid ratio was 2.48 , which was significantly better than the values achieved by other previous methods.

These investigations showed that some physical and chemical properties of fruits and vegetables (including nutrient content, hardness, degree of damage, degree of disease, and degree of maturity) can be revealed through RGB images, sample spectral information and acoustic spectral information. Better prediction and classification effects can be achieved through deep-learning model training. In Table 4, we summarize the technical details of the studies mentioned in this subsection.

\section{Detection of biotic/abiotic stress in horticultural crops}

Singh et al. ${ }^{6}$ reviewed the application of deep-learning methods to plant stress phenotypes in 2018. According to their summary, deep learning can be applied to the identification, classification, quantification, and prediction (also called the ICQP paradigm) of plant stress phenotyping $^{6}$. In this section, we comprehensively outline the publications that employ deep learning for the stress phenotyping of horticultural plants (Table 5). The traditional identification and classification of plant stress have always relied on the recognition of visual symptoms by human experts as a means of categorization, which is inevitably subjective and error prone ${ }^{90}$. Computer vision coupled with machine-learning technology has the capability of automatic identification and classification and enables accurate, scalable and high-throughput phenotyping. Among the machine-learning methods, deep learning has been considered one of the most effective approaches for improving the overall performance of object detection and recognition processes ${ }^{6,91}$.

To identify various biotic and abiotic stresses in tomatoes, efforts have been made with different kinds of deeplearning approaches. Fuentes et al. ${ }^{91}$ combined three deep-learning meta-architectures, namely the Faster R-
$\mathrm{CNN}$, region-based FCN (R-FCN), and SSD, with two deep feature extractors (ResNet and VGGNet) to detect pests and diseases in tomatoes. To detect diseases in tomatoes, Karthik et al. ${ }^{92}$ proposed a model to apply an attention gating mechanism in a residual $\mathrm{CNN}$ with the PlantVillage dataset, which contains three kinds of diseases in tomatoes, namely, leaf mold, early blight, and late blight, for disease detection. An overall accuracy of $98 \%$ was achieved with the proposed model on the validation datasets by adopting a fivefold cross-validation method, in which the original sample was randomly divided into five subsets of equal size and one subset was used as the validation data and the other four for training the model. The cross-validation process was then repeated five times, and the results from the five iterations were averaged (or otherwise combined) to produce a single estimation. In another study, images of tomatoes were acquired from the open PlantVillage database; the images included various bacterial (bacterial spot), viral (tomato mosaic virus and yellow leaf curl virus), and fungal (leaf mold, target spot, early blight, and late blight) diseases and pests (such as spider mites). The proposed framework reached an accuracy of $99.18 \%$ with GoogLeNet, while AlexNet had an accuracy of $98.66 \%{ }^{46}$.

For the identification of various diseases in cucumbers (diseases such as anthracnose, powdery mildew, downy mildew, gray mold, target leaf spots and black spot), a deep-learning approach was also used. Lin et al. ${ }^{35}$ presented a semantic segmentation method based on a CNN to identify powdery mildew on cucumber leaves and achieved the following results: the average pixel accuracy was $96.08 \%$, the Dice accuracy was $83.45 \%$ and the intersection over union was $72.11 \%$ (the source code is available at https:/github.com/ChrisLinSJTU/ segmentation-of-powdery-mildew) ${ }^{35}$. In another study on a recognition model for cucumber diseases, the DCNN model achieved an accuracy of $93.4 \%{ }^{93}$.

In another application, AlexNet and VGG-16 were proposed to classify five eggplant diseases (little leaf, epilachna beetle infestation, cercospora leaf spot, tobacco mosaic virus (TMV) and two-spotted spider mite) and healthy plants with images acquired from smartphones. They used the modified VGG-16 model to achieve an accuracy of $93.33 \%{ }^{94}$. Another novel deep-learning architecture with a global pooling dilated CNN (GPDCNN) was presented for cucumber leaf disease (gray mold, powdery mildew, anthracnose, downy mildew, black spot, and angular leaf spot) recognition by the combination of a dilated convolutional neural network with global pooling ${ }^{95}$. The results showed that GPDCNN had a higher recognition accuracy and shorter training time than the DCNNs and AlexNet ${ }^{95}$.

In a recent study, an image-based field monitoring system combined with a weakly supervised training 


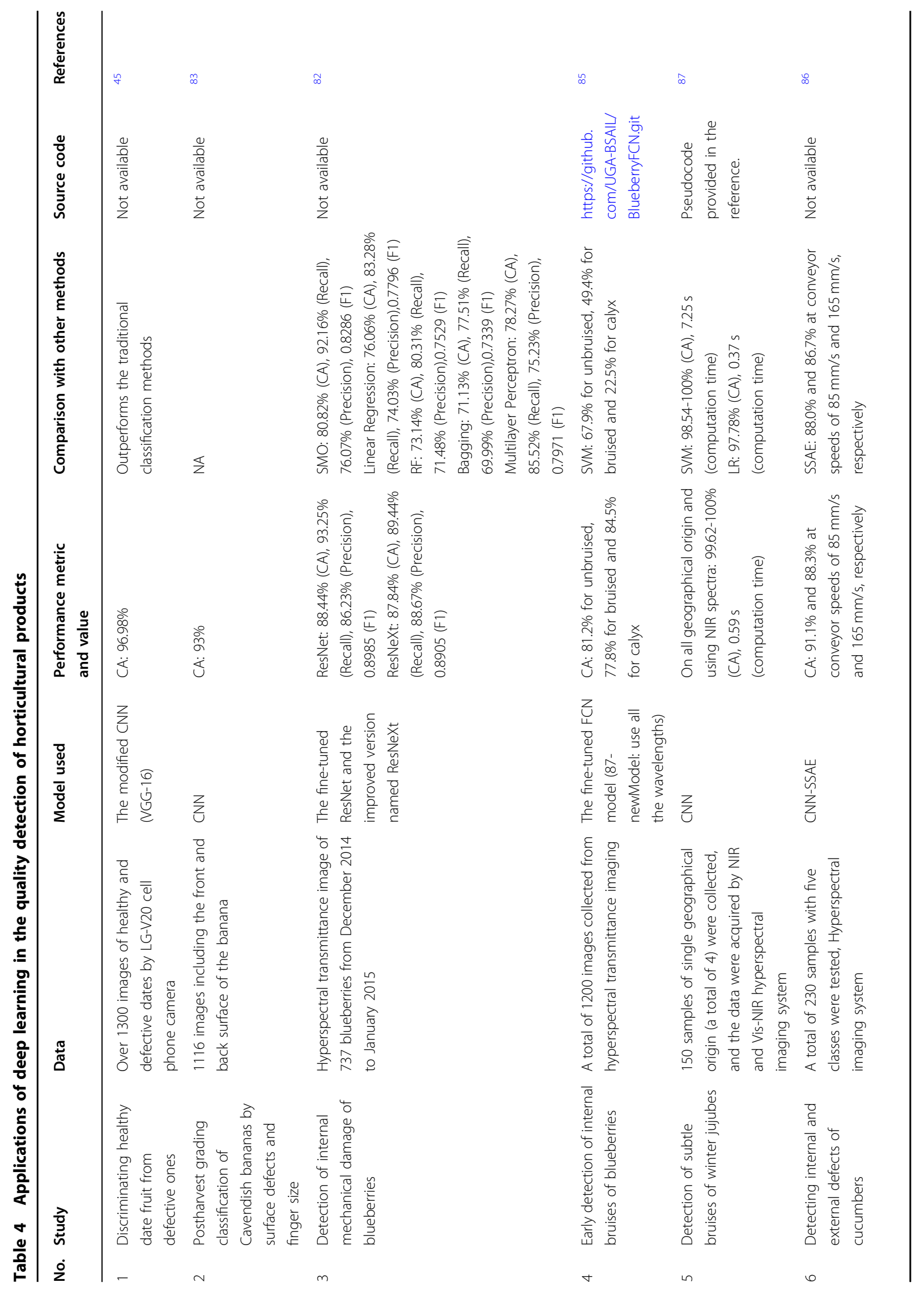




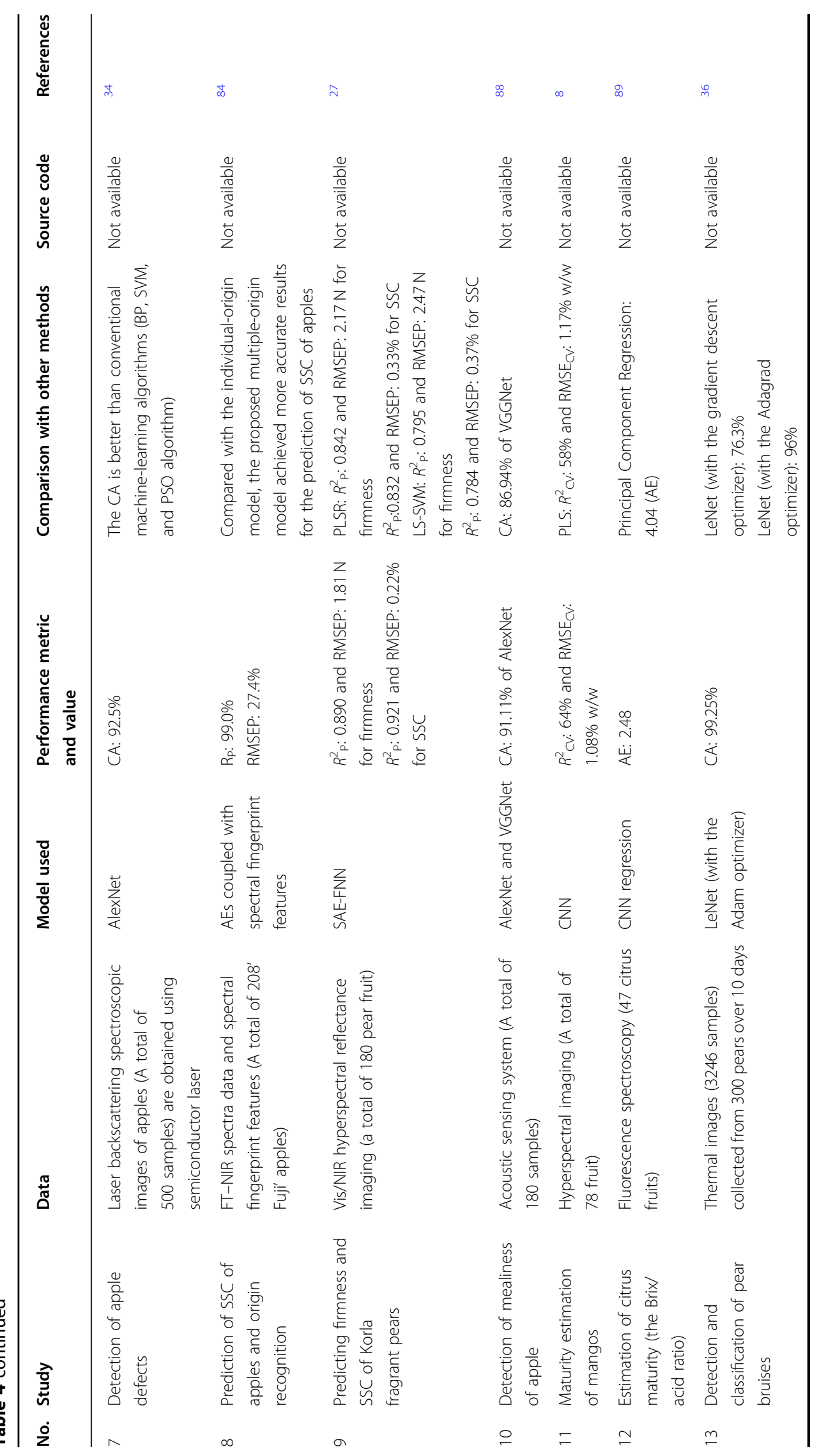




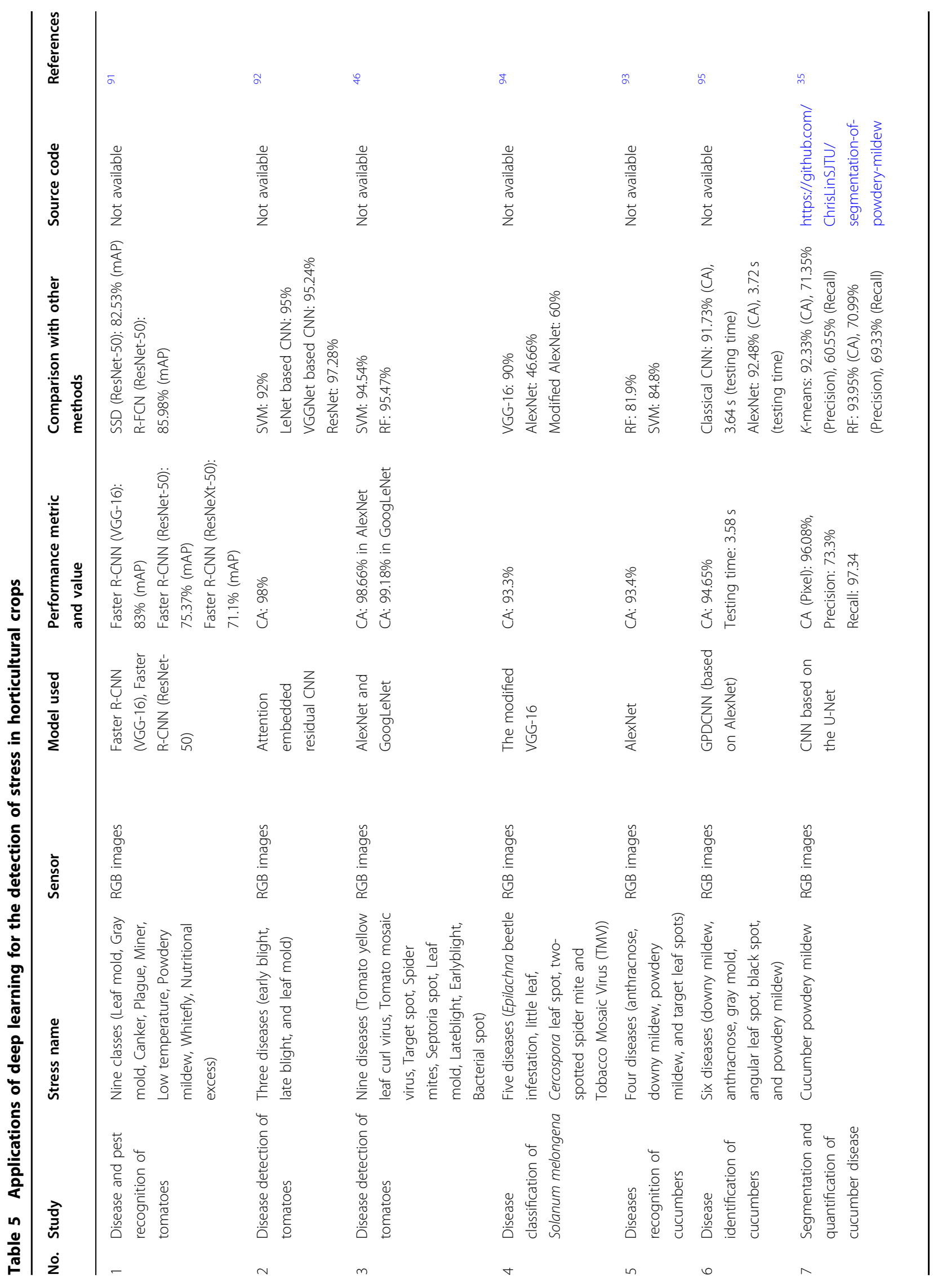




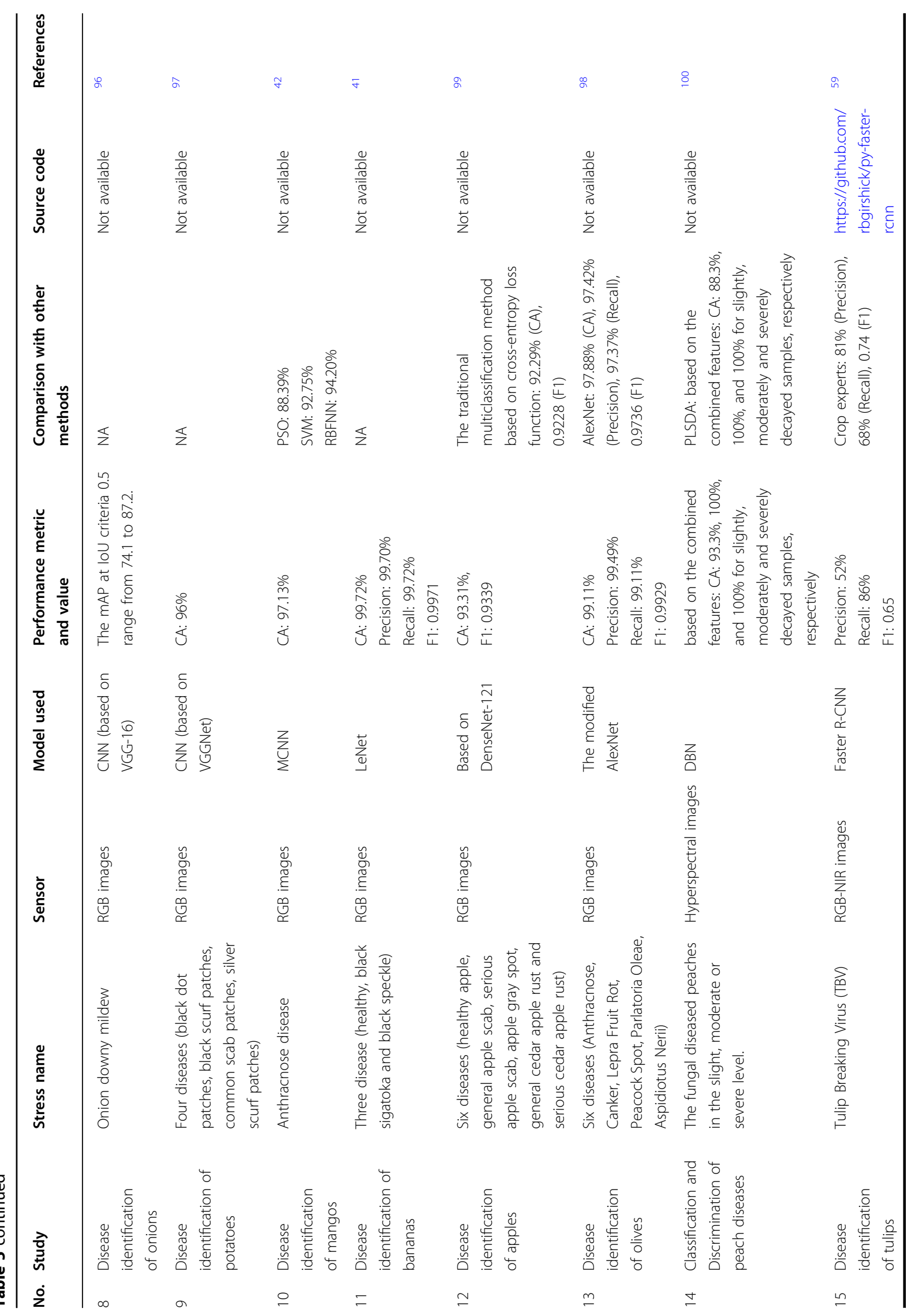


method was developed for automatic onion disease detection and growth monitoring in real-time. The results showed that the mAP (mean Average Precision) score at an IoU (Intersection of Union) criteria of 50\%, which indicates a $50 \%$ overlap, was the highest among all the existing models and was between 74.1 and $87.2^{96}$. In another study, a CNN was trained to classify diseased potato tubers into five classes, including four disease classes and a healthy class, with an accuracy of $96 \%{ }^{97}$. Alruwaili et al. $^{98}$ proposed an enhanced CNN model named AlexNet for detecting and classifying olive diseases. The proposed method achieved overall accuracy, recall, precision, and $\mathrm{F} 1$ scores of $99.11 \%, 99.11 \%, 99.49 \%$, and $0.9929 \%$, respectively. In another application, three methods, including regression, multilabel classification and a focus loss function based on the DenseNet-121 DCNN, were proposed to detect diseases on apple leaves ${ }^{99}$. The proposed three methods obtained accuracies of $93.5 \%, 93.3 \%$, and $93.7 \%$ on the test dataset, which are better than those obtained by the traditional multiclassification approach.

With the goal of automating disease identification and classification, a multilayer CNN (MCNN) was proposed for classifying mango leaves infected by anthracnose fungal disease with an accuracy of $97.13 \%{ }^{42}$. The LeNet architecture was applied to classify and identify banana leaf diseases ${ }^{41}$. The Faster R-CNN architecture was proposed to automatically detect the Tulip Breaking Virus (TBV) and reached an efficiency of $0.13 \mathrm{~s}$ per image ${ }^{59}$. A deep belief network (DBN) model based on 494 features was developed to classify peach samples with slightly decayed, moderately decayed and severely decayed diseases. The results showed that the highest classification accuracies for the three kinds of peach diseases mentioned above were $82.5 \%, 92.5 \%$, and $100 \%$, respectively ${ }^{100}$.

The early and accurate detection of plant diseases is considered an effective method to maintain and improve crop quality and minimize production losses. As a result, deep-learning approaches have received wide recognition worldwide because of their accurate and efficient detection of plant diseases in the field. In Table 5, we summarize the technical details of the studies mentioned in this subsection.

\section{Growth monitoring in horticultural crops}

Crop traits are important to plant breeders and producers for plant production management, as well as for making intelligent decisions about excellent genotype selection when yield traits or quality traits are used. The automatic intelligent collection of horticultural crop growth information in advance provides a good basis for planters to monitor growth and plan the harvesting timeline during the maturation of fruits and vegetables ${ }^{37,48}$. Lu and his team have proposed methods to localize mushrooms and track their growth ${ }^{37,57}$. Lu et al. $^{57}$ adopted the YOLO algorithm to localize mushrooms in an image and proposed a positioning correction method to modify the localization result. After the mushrooms had been precisely localized, $\mathrm{Lu}$ et al. $^{37}$ developed an image measurement system to record the diameter of the mushroom caps during the maturation period. The proposed algorithm (the YOLO-V3 + SP algorithm) was used to calculate the mushroom circles based on the images captured by a camera continuously and then to record the growth information of the mushroom caps; the method outperformed the current circle Hough transform method (OpenCV's implementation) ${ }^{37}$.

Automatic detection and identification of fruits and flowers at various growth stages is important for the automatic and intelligent management of orchards. Tian et al. ${ }^{101}$ proposed an instance segmentation model by improving the mask scoring R-CNN with a U-Net backbone (MASU R-CNN) to detect and segment apple flowers at three different stages: bud, semiopen and fully open. Furthermore, Tian et al. ${ }^{102}$ proposed an improved YOLO-V3 model to detect apples at various growth stages, i.e., young, expanding, and ripe apples, in orchards with complex backgrounds. The detection performance of the proposed YOLO-V3-dense model was better than that of the original YOLO-V3 and the Faster R-CNN with VGG-16 net models. Wang et al. ${ }^{48}$ also developed an automated growth monitoring system in an apple orchard to monitor apple growth during the period of fruit thinning and fruit ripening. They used the fused convolutional features (FCF) model to segment apple images. The mean average absolute error of an apples' horizontal diameter obtained by the method was $0.90 \mathrm{~mm}$. In another study, Tu et al. ${ }^{44}$ developed a machine vision model for detecting passion fruits and identifying their maturity by utilizing natural outdoor RGB-D images combined with the DSIFT (dense scale invariant features transform) algorithm and the LLC (locality-constrained linear coding) method. Finally, the features collected by RGB-DSIFT-LLC were fed into a SVM classifier for the identification of fruit maturity at five different levels: young $(\mathrm{Y})$, near-young $(\mathrm{NY})$, near-mature $(\mathrm{NM})$, mature $(\mathrm{M})$, and after-mature (AM). The proposed method achieved an accuracy of 92.7\% for detection and $91.5 \%$ for maturity classification. $\mathrm{Ni}$ et $\mathrm{al}^{38}$ adopted another deep-learning method based on the Mask R-CNN model and an iterative annotation strategy to detect and segment blueberry fruit to monitor the maturity of the blueberry fruit. The proposed model obtained reasonable prediction performance, with a coefficient of determination $\left(R^{2}\right)$ for the detected berry number with respect to the ground truth of $88.6 \%$ and a RMSE of 1.484 . 
It is important to recognize the different sizes of the panicle-associated image area and the number of panicles as indices of flowering. Wang et al. ${ }^{103}$ developed a machine vision assessment system to detect flower panicles on mango plants at different stages of growth, from green to light yellow, light pink, and then brown-red, which correspond to panicle development ranging from the early to late stages. In another study, Koirala et al. ${ }^{104}$ proposed four architectures based on a deep-learning method for mango panicle stage classification at three different stages: panicles with flowers not fully opened (whitish in color), panicles with opened flowers and panicles displaying flower drop and fruit set. While the YOLO-V3-rotated model had a higher accuracy in terms of the total number of panicles, the $R^{2} \mathrm{CNN}$-upright model was superior for the classification of panicle stages (the source code for the $R^{2} \mathrm{CNN}$ method is available at: https://github.com/DetectionTeamUCAS/R2CNN_Faster-

RCNN_Tensorflow) ${ }^{104}$. To extract growth and quantity information from the large-scale aerial images collected from a lettuce field, Bauer et al. ${ }^{105}$ proposed the AirSurfLettuce platform, which applied a CNN-based-learning model (the source code is available at https:/github.com/ Crop-Phenomics-Group/AirSurf-Lettuce). The AirSurfLettuce can automatically measure in-field iceberg lettuces with a focus on yield-related traits, such as field size distribution, lettuce size categories, number of plants, and GPStagged harvest regions, and has great potential to support smart and precise crop surveillance.

From the investigations given above, it is known that deep-learning methods have been applied to growth monitoring in horticultural crops and have achieved better prediction and classification effects, the technical details of which are summarized in Table 6.

\section{Other applications}

Genomic prediction (GP) is the process in which untested good genetic attributes are predicted by employing genome-wide marker information ${ }^{40}$. Recently, deep-learning technologies have been applied as powerful machine-learning tools to quantitatively predict phenotypes without intrudation to analyze the increasing amount of available genetic and genomic data. Although numerous examples of GP have been widely utilized to improve the breeding efficiency of plants and animals ${ }^{40,106}$, applications to horticultural crops are still in the preliminary stage. Zingaretti et al. ${ }^{40}$ evaluated the genomic prediction performance of a deep-learning method for two common and important horticultural fruits: the autotetraploid blueberry and allooctoploid strawberry. The two datasets included a total of 1802 autopolyploid blueberry $(2 n=4 x=48)$ and 1358 allopolyploid strawberry $(2 n=8 x=112)$ individuals genotyped to create 73,045 and 9908 single-nucleotide polymorphism (SNP) markers, respectively, and phenotyped by five distinctive agronomic traits, including fruit size, firmness, picking scars, weight, and yield. A potential superiority of deep learning for GP over some standard linear approaches is that deep learning can potentially consider all possible genetic interactions, including epistasis and dominance, which are considered to be particularly relevant in most polyploids.

The sale of horticultural products is an essential part of the product supply chain. Owing to the asymmetry between a farmer's production and the real marketing information and the asymmetry between the social signal of product supply and demand, the prices of horticultural products fluctuate greatly. Therefore, it is very important to forecast the prices of horticultural products when creating a planting plan $^{39}$. Weng et al. ${ }^{39}$ adopted the autoregressive integrated moving average (ARIMA), BP and RNN methods to forecast the daily, weekly, and monthly average prices of different horticultural products (cucumbers, tomatoes, and eggplants). With web crawler technology, a large amount of data on horticultural product prices were gathered from the website. The results indicated that neural network methods including the BP and RNN methods had a higher accuracy in price forecasting than that of the ARIMA model. They considered that deep-learning methods would serve as the mainstream method for price forecasting of horticultural products in the near future.

\section{The advantages and disadvantages of deep- learning technology}

The most notable advantages of deep-learning technologies lie in their automatic feature extraction, classification and prediction processes ${ }^{7}$. The handcrafted extraction of features and the design of feature descriptors are generally very difficult and time consuming and are no longer required for deep-learning technologies through automated feature extraction. Generally, the prediction accuracy of deep-learning models improves as the number of model layers increases, which is accompanied by increased computational complexity. In addition, it is not an easy task to build a good feature extractor. In fact, a great deal of the aforementioned studies for the prediction and classification of horticultural plants, including flowers or fruits, used existing models or made only a minor adjustment to them, and the main contributors were also mostly scholars from computer science and image processing sectors. Therefore, higher attention was given to the techniques for general feature extraction and classification of images rather than the specific features of a horticultural product. For instance, in the applications of crop yield estimation, the manual extraction of features that may significantly affect crop growth was nearly impossible. Thus, it is important to design deep-learning models that specifically focus on such feature extraction 


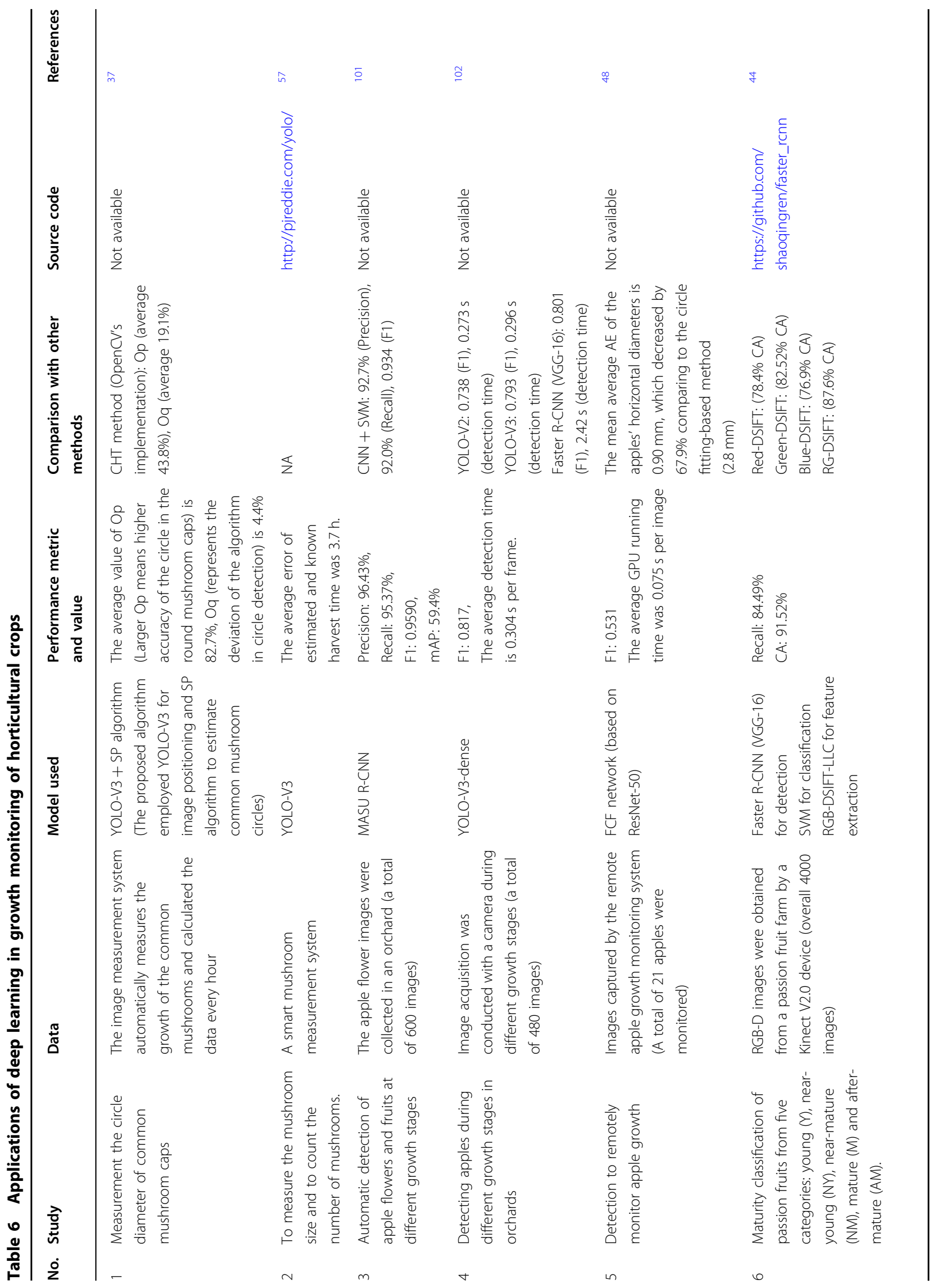




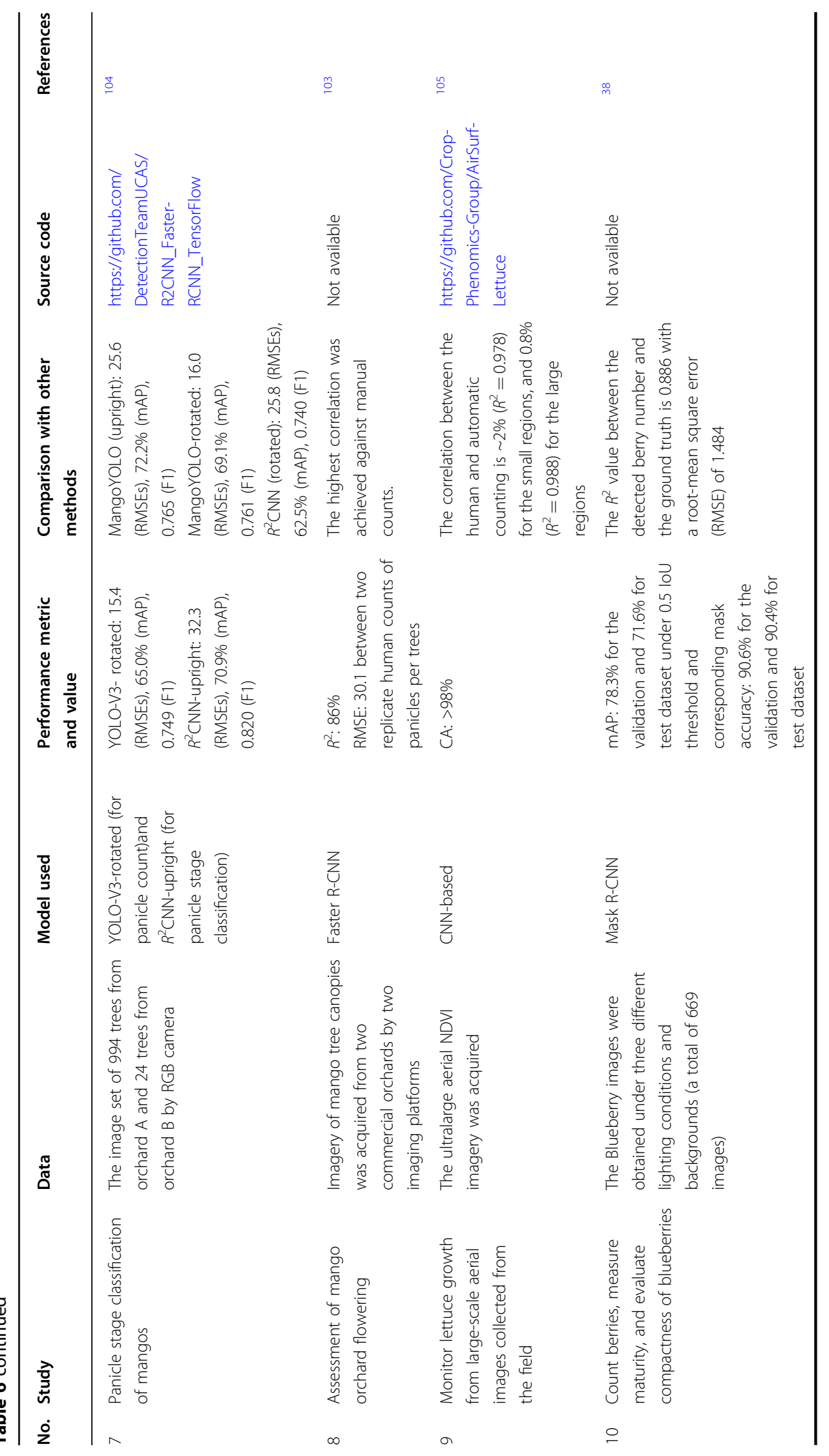


processes to more efficiently and cost-effectively apply deep-learning models to horticultural sciences. Fortunately, deep learning has another useful characteristic: its transfer learning ability ${ }^{28}$. With this technique, researchers can make use of the existing models already trained by a large amount of source data to solve similar problems. By doing so, they generally need to adjust some layers and use only the target data (the data they are going to learn) to fine-tune the already trained model. Through fine-tuning, the efficiency and performance in modeling subsequent tasks is improved. For example, in the studies mentioned above, some examples exploited predesigned networks (e.g., VGGNet or AlexNet) based on a large dataset (such as ImageNet) and applied them to their specific learning task that required a much smaller dataset through fine-tuning to achieve better-than-before results.

As deep-learning contains more complex model structures and requires higher computational efforts, its development and applications are somewhat limited for noncomputer experts ${ }^{7}$. Fortunately, contributors, such as computer scientists and deep-learning enthusiasts, around the world have developed many software and hardware tools to help nonexpert researchers to easily and quickly develop deep-learning technology. For software support, some popular models and their variations have been designed to reduce programming difficulties so that nonexpert researchers can build required networks quickly. The aforementioned models, such as AlexNet, CNN-SSAE, DaSNet, LedNet, and VGGNet, are just a few. Apart from these ready-to-use models, there are also a number of websites from which researchers in horticulture sectors can learn to improve their deep-learning skills and build their own models. For example, https:// www.fast.ai/ provides online courses that are free and have no ads, https://www.deeplearning.ai/ provides specialized deep-learning techniques; AI for everyone, TensorFlow specialization, and https://mlcourse.ai/ provide open machine-learning courses. For hardware support, a GPU combined with the compute unified device architecture (CUDA) toolkit developed by NVIDIA is a good candidate to accelerate deep-learning computation. There is also some specialized hardware designed for accelerating deep-learning processes, among which the Tensor Processing Unit (TPU) developed by Google, the AI Processors, Vision Processing Units (VPUs) and the Neural Compute Stick 2 (NCS 2) by Intel, the Efficient Inference Engine (EIE) by Stanford, and the EnergyEfficient Reconfigurable Accelerator for DCNNs (Eyeriss) by MIT are some useful tools. The proposed network frameworks and hardware acceleration tools can be used together, can greatly reduce the computational time and have the potential to perform prediction and/or classification well to meet the needs of real-time applications in data processing.

Nevertheless, it cannot be denied that deep learning has its own shortcomings. First, the optimization tasks are sometimes quite complicated and very time consuming due to the large datasets and large numbers of weights to be tuned; additionally, there are some hardware restrictions and numerous hyperparameters, which are highly complex, that need to be tuned in the models mentioned in Section "Brief overview of deep learning". Furthermore, to replicate a given study and compare it with others, the source codes/algorithms and the model parameters must be reported, and the evaluation metrics for the performance measures should be standardized. From the reported studies, regarding the methods used in the different research works, various performance metrics have been employed by the authors. For example, we noted that performance metrics, such as accuracy, precision, recall, F1-score, mAP, RMSE and IoU, were employed in the literature to report model performance. However, to compare a reported model and to improve model performance in the existing studies, the use of performance metrics should be unified and standardized in future research. Additionally, different models, learning algorithms, hyperparameters or validation processes have been applied and vary from species to species or object to object. Such specificity limits the widespread application of the proposed technologies outside of the given research domain. Further work should be done to standardize the proposed technologies and overcome the bottleneck to build robust and easy-to-use models. Noncomputer experts, such as horticulture scientists, will be able to find and use such user-friendly models for practical applications in the future.

Another limitation lies in the fact that the collection of a large and reliable dataset along with clear data annotation is inevitably tedious work, which make completing tasks more complicated for researchers. The success of deep learning cannot be realized without the availability of annotated data ${ }^{107}$. Data collection and annotation is crucial and time consuming; therefore, data collection efforts should be made by researchers worldwide. Moreover, since the collection ability of data by individuals, research teams, or even institutions is limited, the collected data should be uploaded to open-access databases to lower the entry barrier and to accelerate the availability of of data for researchers. On the other hand, a number of ongoing examples of code sharing are now available on GitHub and other Git-like platforms developed by some enthusiasts for nonexperts to share models and check data ${ }^{1}$. Therefore, a combined effort by both horticulture and computer scientists is necessary to make significant 
contributions to meet challenges in intelligent horticulture fields.

\section{Summary, discussions, and future perspectives Summary and discussions}

Through a careful examination of existing studies, we found that the major research focus was on the development of deep-learning models and their potential applications in various horticultural studies. The application of deep-learning techniques in horticultural sectors is still in its nascent stage but is also in a period of rapid development. From 2016 until now, there have been 71 relevant publications focusing on the applications of deeplearning methods to species identification and variety classification $(33.80 \%)$, quality detection (18.31\%), yield prediction $(9.86 \%)$, pest and disease management (21.13\%), and growth monitoring (14.08\%) in the field of horticultural crops in addition to some review papers (2.82\%). Among the surveyed papers, the number of publications in 2019 was $130 \%$ more than that in 2018 (30 in 2019 versus 13 in 2018). Since most of the works were based on image processing, CNNs and their variants were chosen in most cases $(92.96 \%$ among the surveyed literature). From our reviewed work (Tables 2-6), 56 out of the 71 papers $(78.87 \%)$ performed valid, correct and direct comparisons between the proposed approach and other state-of-the-art techniques with respect to the same problem. The results of these studies indicate that deep learning has better performance in many aspects compared to traditional machine learning for many agricultural tasks ${ }^{23}$.

In terms of evaluating model performance, various performance metrics were employed by the original author(s), and each metric was associated with the model used in each study. At the end of Section "Brief overview of deep learning", some performance metrics, their definitions/descriptions, and the abbreviations we used in this paper are described. For some studies, where the author(s) directly used accuracy/correct recognition rate without providing its definition, we assumed that they referred to CA. From Tables 2-6, we can see that CA was the most popular metric used (43 papers, $60.56 \%$ ), followed by F1 (18 papers, 25.35\%). Some papers included the RMSE (8 papers), $R^{2}$ (3 papers) or other metrics. Twenty-seven of the 71 studies $(38.03 \%)$ used a combination of performance metrics to evaluate their results. Usually, in combination with CA, F1, precision, recall, or IoU was also used to evaluate the prediction performance of the models.

As each paper adopted different datasets, performance metrics, preprocessing techniques, models and parameters, it is difficult to compare and generalize the results from different papers. Therefore, our comparisons and generalizations would generally be strictly limited to the results from a single paper. However, in this section, we still tried to make some evaluations and comparisons among different technologies, but the readers should take our comments with caution, as the datasets and other parameters used by different authors might not be the same. In 43 of the 71 papers that used CA as a metric, most of the accuracy was higher than $90 \%$, indicating good performance. The highest CA results had values higher than $98 \%$, constituting remarkable results, which were obtained by Amara et al. ${ }^{41}$ (99.72\% with a LeNet model), Feng et al. ${ }^{87}$ (99.62-100\% with a CNN), Zeng et al. $^{36}$ (99.25\% with a modified LeNet model), Brahimi et al. ${ }^{46}$ (99.18\% with GoogLeNet and $98.66 \%$ with AlexNet), Alruwaili et al. ${ }^{98}$ (99.11\% with a modified AlexNet model), Neupane et al. ${ }^{78}$ (98.7\% with the Faster R-CNN Inception-V2 model), Giefer et al $^{56}$ (98.36\% with the VGG-16 model), Karthik et al. ${ }^{92}$ (98\% with a residual $\mathrm{CNN}$ ), Bauer et al. ${ }^{105}$ (higher than $98 \%$ with a $\mathrm{CNN}$ ), and Sun et al. ${ }^{100}(93.3 \%-100 \%$ with a DBN). Of the surveyed papers, the results obtained by Zhang et al. ${ }^{85}$ had the lowest CA (77.8\%-84.5\% with an FCN model); however, the SVM model used in this particular task (the detection of internal bruises in blueberries) also obtained a low CA (22.5\%-67.9\%). Additionally, in Zhu et al. ${ }^{30}$, the AlexNet model (92.1\%) obtained CA results that were $14.1 \%$ and $11.6 \%$ better than the results of the BP neural network (78\%) and SVM (80.5\%), showing a significant improvement. On the other hand, among the 43 papers that used $\mathrm{CA}$ as the metric, AlexNet was the most adopted model (10 papers, $23.2 \%$ ), followed by VGGNet (8 papers, $18.6 \%$ ) and ResNet (5 papers, 11.6\%). From the 18 papers that used F1 as a metric, 6 papers obtained values above 0.90 and the highest values, which were observed by Ampatzidis et al. ${ }^{72}$ (0.998 with the YOLO-V3 model), Amara et al. ${ }^{41}$ (0.9971 with the LeNet model), and Alruwaili et al ${ }^{98}$ (0.9929 with a modified AlexNet model), were above 0.99 , indicating excellent performance. The results obtained by Wang et al. ${ }^{48}$ had the lowest F1 score $(0.531$ with an FCF model), but the average running time in the GPU was greatly reduced and was $0.075 \mathrm{~s}$ per image.

\section{Future perspectives of deep learning in the horticulture domain}

With the progress of scientific research, deep-learning methods and applications will have a great impact on the horticultural industry and the potential to overcome various challenges (such as productivity challenges, environmental changes, food security and sustainability) in the agricultural industry ${ }^{5,16,108}$. Tables 2-6, which lists a variety of existing applications of deep-learning methods in horticultural science, show that attempts to classify species, detect quality, predict yield and manage pests have been implemented. For example, precise fruit/ flower/vegetable detection with deep-learning technology 
allows the generation of yield maps to provide real-time information on spatial variation from which agronomic decisions can be based to form efficient and precise harvesting strategies for increasing marketable yield ${ }^{2,105}$. This technology would take the place of farmers or gardeners and could solve the current problem of relying heavily on personal experience, which is time consuming, inaccurate and unreliable. Another promising example is the early detection of plant stress ${ }^{6,90}$. Combining deep learning with digital image data or spectral data has shown great potential in improving the speed, accuracy, reliability and scalability of early detection, classification and quantification of plant stress and/or disease ${ }^{6,16}$. In addition, because of the availability of inexpensive digital imaging devices, IoT capabilities, and computing and data storage capabilities, more varieties of horticultural crop information can be used to train deep-learning models and to address some valuable specific issues in the horticultural field.

Although deep learning has superior performance in most of the studies, it is not easy for a reader to quickly choose the right model for a specific task. This difficulty comes not only from the selection of a deep-learning model but also from the hardware and software conditions, weight initialization, learning algorithms, learning rates, activation functions, hyperparameters, validation processes, data sources and data preprocessing methods. Therefore, deep learning can be considered more of an art that relies heavily on personal experience than science. If an established model is to be widely applied to ordinary farms and orchards, it would be necessary to accept that the detection accuracy may be reduced. Since most of the studies used deep learning for image object detection, a CNN was chosen to be successfully applied for the recognition and yield estimation of horticultural plants/products.

There are three main types of CNN-based object detection methods. The first includes some main CNN structures for object recognition, such as $\operatorname{LeNet}^{36,41}$, AlexNet $^{30,62,63}$, VGGNet ${ }^{43,91}$, GoogLeNet ${ }^{46}$, and $\operatorname{ResNet}^{31,48}$. On the basis of the first type, the second type of method realizes two-stage deep-learning object detection combined with the region proposal method to achieve an improvement in the detection rate and an acceleration in the detection speed; this method type mainly includes the $\mathrm{R}-\mathrm{CNN}^{33,73}$, Faster $\mathrm{R}-\mathrm{CNN}^{44,59,60,77}$ and Mask R-CNN ${ }^{38,54}$. The third type includes end-toend, single-stage deep-learning object detection algorithms, which can directly return the categories and position borders of multiple objects, such as the YOLO $^{37,72,74}$ and SSD ${ }^{56}$ methods. Based on these models, fruit yield can be automatically estimated ${ }^{32,54,68}$, flower and fruitlet thinning and other gardening operations can be automatically conducted ${ }^{48,101}$, and the early detection of plant stress can be accomplished ${ }^{6,90}$.
However, for the quality detection of horticultural products and stress detection of horticultural plants, such as some invisible quality indicators or early bruising detection, a visual analysis method is less effective. From Tables 4 and 5 , the authors not only adopted regular CNN methods but also employed other networks, such as the SAE-FNN ${ }^{27}, \mathrm{CNN} \mathrm{SSAE}^{86}, \mathrm{DBN}^{100}$ methods, to achieve accurate and rapid feature extraction from a large number of hyperspectral image data and to detect fruit quality (such as SSC and hardness) and plant stress (such as early bruising detection and disease identification). In addition, as Vis/NIR hyperspectral imaging may allow for early detection of plant stress/internal bruising of fruit before symptoms are visible to human eyes, deep-learning approaches would also be promising in this domain. Therefore, deep-learning technology should be combined with these rapid nondestructive testing technologies to explore its great potential in effective feature extraction for direct defect detection. In addition, RNN or LSTM models combined with high-performance regression algorithms or classifiers are promising options that can be used in future horticultural research, especially in yield and price predictions of horticultural products. One example was given in this review that applied LSTM to forecast a banana harvest yield for effective and efficient decision making and forward planning ${ }^{32}$.

Moreover, owing to the advancement of hardware computing capabilities and hardware supply, training processes can be accomplished in much shorter amounts of time. We believe that with the commercialization and widespread use of the Qualcomm Neural Processing SDK (Software Development Kit) for AI and other mobile platforms, handheld smart devices and mobile deeplearning applications will be available for ordinary farms and orchards in the near future. Some of the successful cases deploying deep-learning concepts for plant science applications, such as image recognition and the quality evaluation of horticultural products, early detection systems for plant stress, and yield prediction, can be further transformed for practical application in horticulture ${ }^{5,16,23}$. According to the surveyed papers, some authors ${ }^{20,58,109}$ further integrated tree trunk, branch or fruit recognition algorithms into mobile devices after the model was trained. However, to truly achieve deep learning on mobile devices, some difficulties and problems need to be addressed, such as how to embed a model into mobile devices and how to realize the miniaturization of sensing equipment. Thus, more research is needed to achieve this goal.

Currently, deep-learning technology has been able to open the door to intelligent horticulture for difficult gardening jobs. Based on these precise phenotypic data, we can accurately monitor crop growth features at different critical stages and key yield-related traits and 
implement precise agricultural decision management. As shown in Table 1, the performance of deep learning combined with image data has steadily improved, and these models have also been successfully applied in horticultural science. However, as was described before, it is a difficult task to choose the most suitable model among the various techniques that have been proposed to date for a specific application in horticultural science. In addition, there is also an urgent need to build large datasets containing plant images to create robust models. When collecting plant phenotypic data, we strongly recommend the use of in-field real condition imaging data (i.e., with varying shade, light, and mutual occlusion conditions) to create training datasets. Correct labeling and the open-source use of these datasets can avoid the duplication of data collection. With the increasing amount of collaborative research and the joint effort among horticulturalists and computer scientists, we are confident that deep-learning technology has great potential to support the horticulture industry more intelligently and accurately to improve yield and quality and to better detect plant stress and diseases.

\section{Conclusion}

With the rapid explosion of data in horticultural sciences, deep-learning technology has become a hot research focus and has opened a new area in artificial intelligence. Deep-learning methods provide a powerful tool to assimilate data and have proven to hold promise for overcoming the existing challenges to record plant growth objectively, judge plant status accurately and detect the quality of products quickly in horticultural science. A key element for the successful large-scale application of deep-learning technology lies in the joint effort of scientists from both computer and horticulture sectors and the seamless integration of data collection along with an effective curation pipeline ${ }^{14}$. Such efforts would allow for the formation of a computational ecosystem that might provide tremendous opportunities to facilitate planting, promote intelligent orchard management and tackle other problems. Some of the solutions discussed in this paper also have potential for commercialization in the near future. For example, automatic robots incorporated with a faster region-based CNN could be used in transplanting, fruit picking or yield estimation. The aim of this review is to introduce this relatively new and effective tool so that researchers and workers in horticulture sectors can manage the massive amounts of data they might collect in their research and to encourage researchers to use or improve data to solve their problems to gradually to move towards a smart horticulture industry.

\section{Acknowledgements}

This study was financially supported by the Fuzhou Science and Technology Major Project, grant number 2018NZ0002-2 and the Science and Technology Innovation Special Fund Project of Fujian Agricultural and Forestry University, grant number CXZX2018032.

\section{Conflict of interest}

The authors declare no competing interests.

Received: 3 August 2020 Revised: 13 March 2021 Accepted: 22 March 2021 Published online: 01 June 2021

\section{References}

1. Nturambirwe, J. F. I. \& Opara, U. L. Machine learning applications to nondestructive defect detection in horticultural products. Biosyst. Eng. 189, 60-83 (2020).

2. Chen, F. et al. Genome sequences of horticultural plants: past, present, and future. Hortic. Res. 6, https://doi.org/10.1038/s41438-019-0195-6 (2019).

3. Colaço, A. F., Molin, J. P., Rosell-Polo, J. R., \& Escolà A. Application of light detection and ranging and ultrasonic sensors to high-throughput phenotyping and precision horticulture: current status and challenges. Hortic. Res. $\mathbf{5}$ https://doi.org/10.1038/s41438-018-0043-0 (2018).

4. Edwards, E. J., \& Moghadam, P. Intelligent systems for commercial application in perennial horticulture. Proceedings 36, 59 (2020).

5. Kamilaris, A. \& Prenafeta-Boldú, F. X. A review of the use of convolutional neural networks in agriculture. J. Agric Sci. 156, 312-322 (2018).

6. Singh, A. K, Ganapathysubramanian, B., Sarkar, S. \& Singh, A. Deep learning for plant stress phenotyping: trends and future perspectives. Trends Plant Sci. 23, 883-898 (2018).

7. Zhou, L., Zhang, C., Liu, F., Qiu, Z. \& He, Y. Application of deep learning in food: a review. Compr. Rev. Food Sci. Food Saf. 18, 1793-1811 (2019).

8. Wendel, A., Underwood, J. \& Walsh, K. Maturity estimation of mangoes using hyperspectral imaging from a ground based mobile platform. Comput. Electron. Agric. 155, 298-313 (2018).

9. Pan, L. et al. Detection of cold injury in peaches by hyperspectral reflectance imaging and artificial neural network. Food Chem. 192, 134-141 (2016).

10. Leiva-Valenzuela, G. A. \& Aguilera, J. M. Automatic detection of orientation and diseases in blueberries using image analysis to improve their postharvest storage quality. Food Control 33, 166-173 (2013).

11. Che, W. et al. Pixel based bruise region extraction of apple using Vis-NIR hyperspectral imaging. Comput. Electron. Agric. 146, 12-21 (2018).

12. van Dael, $\mathrm{M}$. et al. A segmentation and classification algorithm for online detection of internal disorders in citrus using X-ray radiographs. Postharvest Biol. Technol. 112, 205-214 (2016).

13. Wrzesień, M., Treder, W., Klamkowski, K. \& Rudnicki, W. R. Prediction of the apple scab using machine learning and simple weather stations. Comput. Electron. Agric. 161, 252-259 (2019).

14. Singh, A., Ganapathysubramanian, B., Singh, A. K. \& Sarkar, S. Machine learning for high-throughput stress phenotyping in plants. Trends Plant Sci. 21, 110-124 (2016).

15. Colmer, J. et al. SeedGerm: a cost-effective phenotyping platform for automated seed imaging and machine-learning based phenotypic analysis of crop seed germination. N. Phytol. 228, 778-793 (2020).

16. Kamilaris, A. \& Prenafeta-Boldú, F. X. Deep learning in agriculture: a survey. Comput. Electron. Agric. 147, 70-90 (2018).

17. Koirala, A., Walsh, K. B., Wang, Z. \& McCarthy, C. Deep learning-method overview and review of use for fruit detection and yield estimation. Comput. Electron. Agric. 162, 219-234 (2019).

18. de Ridder, D., Kroese, F., Evers, C., Adriaanse, M. \& Gillebaart, M. Healthy diet: Health impact, prevalence, correlates, and interventions. Psychol. Heal. 32, 907-941 (2017).

19. Altaheri, H., Alsulaiman, M. \& Muhammad, G. Date fruit classification for robotic harvesting in a natural environment using deep learning. IEEE Access 7, 117115-117133 (2019)

20. Majeed, Y. et al. Deep learning based segmentation for automated training of apple trees on trellis wires. Comput. Electron. Agric. 170, 105277 (2020).

21. Noda, K., Yamaguchi, Y., Nakadai, K., Okuno, H. G. \& Ogata, T. Audio-visual speech recognition using deep learning. Appl. Intell. 42, 722-737 (2015). 
22. Ng, H. W., Nguyen, V. D., Vonikakis, V. \& Winkler S. Deep learning for emotion recognition on small datasets using transfer learning. ICMI 2015-Proc. 2015 ACM Int. Conf. Multimodal Interact. 443-449 (ICMl, 2015).

23. Yang, X. \& Sun, M. A survey on deep learning in crop planting. IOP Conf. Ser. Mater. Sci. Eng. 490, https://doi.org/10.1088/1757-899X/490/6/062053 (2019).

24. Lecun, Y., Bengio, Y. \& Hinton, G. Deep learning. Nature 521, 436-444 (2015)

25. LeCun, Y. \& Bengio, Y. in The Handbook of Brain Theory and Neural Networks. 255-258 (MIT Press, Cambridge, MA, USA, 1998).

26. Schmidhuber, J. Deep learning in neural networks: an overview. Neural Netw. 61, 85-117 (2015).

27. Yu, X., Lu, H. \& Wu, D. Development of deep learning method for predicting firmness and soluble solid content of postharvest Korla fragrant pear using Vis/NIR hyperspectral reflectance imaging. Postharvest Biol. Technol. 141 39-49 (2018).

28. Pan, S. J. \& Yang, Q. A survey on transfer learning. IEEE Trans. Knowl. Data Eng. 22, 1345-1359 (2010)

29. VD Prasad, M. et al. An efficient classification of flower images with convolutional neural networks. Int. J. Eng. Technol. 7, 384 (2017).

30. Zhu, L., Li, Z., Li, C., Wu, J. \& Yue, J. High performance vegetable classification from images based on AlexNet deep learning model. Int. J. Agric Biol. Eng. 11, 190-196 (2018).

31. Liu, Z., Wang, J., Tian, Y. \& Dai, S. Deep learning for image-based largeflowered chrysanthemum cultivar recognition. Plant Methods 15, 1-11. (2019).

32. Rebortera, M. A. \& Fajardo, A. C. An enhanced deep learning approach in forecasting banana harvest yields. Int. J. Adv. Comput. Sci. Appl. 10, 275-280. (2019).

33. Chen, Y. et al. Strawberry yield prediction based on a deep neural network using high-resolution aerial orthoimages. Remote Sens. 11, 1-22. (2019).

34. Wu, A., Zhu, J. \& Ren, T. Detection of apple defect using laser-induced light backscattering imaging and convolutional neural network. Comput. Electr. Eng. 81, https://doi.org/10.1016/j.compeleceng.2019.106454 (2020).

35. Lin, K., Gong, L., Huang, Y., Liu, C. \& Pan, J. Deep learning-based segmentation and quantification of cucumber powdery mildew using convolutional neural network. Front. Plant Sci. 10, 1-10 (2019).

36. Zeng, X., Miao, Y., Ubaid, S., Gao, X. \& Zhuang., S. Detection and classification of bruises of pears based on thermal images. Postharvest. Biol. Technol. 161, 111090 (2020).

37. Lu, C. P. \& Liaw, J. J. A novel image measurement algorithm for common mushroom caps based on convolutional neural network. Comput. Electron. Agric. 171, 105336 (2020)

38. Ni, X., Li, C., Jiang, H. \& Takeda, F. Deep learning image segmentation and extraction of blueberry fruit traits associated with harvestability and yield. Hortic. Res. 7, 110 (2020).

39. Weng, Y. et al. Forecasting horticultural products price using arima model and neural network based on a large-scale data set collected by web crawler. IEEE Trans. Comput. Soc. Syst. 6, 547-553 (2019).

40. Zingaretti, L. M. et al. Exploring deep learning for complex trait genomic prediction in polyploid outcrossing species. Front. Plant Sci. 11, 1-14 (2020).

41. Amara, J., Bouaziz, B. \& Algergawy, A. A deep learning-based approach for banana leaf diseases classification. Lecture Notes in Informatics (LNI). Vol. 266, 79-88 (Gesellschaft für Informatik, 2017).

42. Singh, U. P., Chouhan, S. S., Jain, S. \& Jain, S. Multilayer convolution neura network for the classification of mango leaves infected by anthracnose disease. IEEE Access 7, 43721-43729 (2019).

43. Hiary, H., Saadeh, H., Saadeh, M. \& Yaqub, M. Flower classification using deep convolutional neural networks. IET Comput. Vis. 12, 855-862 (2018).

44. Tu, S. et al. Detection of passion fruits and maturity classification using RedGreen-Blue Depth images. Biosyst. Eng. 175, 156-167 (2018).

45. Nasiri, A., Taheri-Garavand, A. \& Zhang, Y. D. Image-based deep learning automated sorting of date fruit. Postharvest Biol. Technol. 153, 133-141 (2019).

46. Brahimi, M., Boukhalfa, K. \& Moussaoui, A. Deep learning for tomato diseases: classification and symptoms visualization. Appl. Artif. Intell. 31 299-315 (2017)

47. Kang, H. \& Chen, C. Fruit detection, segmentation and 3D visualisation of environments in apple orchards. Comput. Electron. Agric. 171, 105302 (2020).

48. Wang, D. et al. Deep learning approach for apple edge detection to remotely monitor apple growth in orchards. IEEE Access 8, 26911-26925 (2020).
49. Saud, A. S. \& Shakya, S. Analysis of look back period for stock price prediction with RNN variants: a case study on banking sector of NEPSE. Procedia Comput. Sci. 167, 788-798 (2020).

50. Song, E., Soong, F. K. \& Kang, H. G. Effective spectral and excitation modeling techniques for LSTM-RNN-based speech synthesis systems. IEEE/ACM Trans. Audio Speech Lang. Process 25, 2152-2161 (2017).

51. Zhang, S., Yi, J., Tian, Z., Tao, J. \& Bai Y. Rnn-transducer with language bias for end-to-end Mandarin-English code-switching speech recognition. In: 2021 12th International Symposium on Chinese Spoken Language Processing (ISCSLP). http://arxiv.org/abs/2002.08126 (Hong Kong, 2021).

52. Williams, R. J. \& Zipser, D. A learning algorithm for continually running fully recurrent neural networks. Neural Comput. 1, 270-280 (1989).

53. Suk, H. I., Lee, S. W. \& Shen, D. Latent feature representation with stacked auto-encoder for AD/MCl diagnosis. Brain Struct. Funct. 220, 841-859 (2015).

54. Yu, Y., Zhang, K. Yang, L. \& Zhang, D. Fruit detection for strawberry harvesting robot in non-structural environment based on Mask-RCNN. Comput. Electron. Agric. 163, 104846 (2019).

55. Le, T. T., Lin, C. Y. \& Piedad, E. J. Deep learning for noninvasive classification of clustered horticultural crops-a case for banana fruit tiers. Postharvest Biol. Technol. 156, 110922 (2019).

56. Giefer, L. A., Castellanos, J. D. A., Babr M. M., \& Freitag M. Deep learning-based pose estimation of apples for inspection in logistic centers using singleperspective imaging. Processes 7, https://doi.org/10.3390/pr7070424 (2019).

57. Lu, C. P., Liaw, J. J., Wu, T. C. \& Hung, T. F. Development of a mushroom growth measurement system applying deep learning for image recognition. Agronomy 9, https://doi.org/10.3390/agronomy9010032 (2019).

58. Zhang, J. et al. Branch detection for apple trees trained in fruiting wall architecture using depth features and Regions-Convolutional Neural Network (R-CNN). Comput. Electron. Agric. 155, 386-393 (2018).

59. Polder, G. et al. Automatic detection of tulip breaking virus (TBV) using a deep convolutional neural network. IFAC-PapersOnLine 52, 12-17 (2019).

60. Hu, C., Liu, X., Pan, Z. \& Li, P. Automatic detection of single ripe tomato on plant combining faster R-CNN and intuitionistic fuzzy set. IEEE Access 7, 154683-154696 (2019).

61. Cibuk, M., Budak, U., Guo, Y., Cevdet Ince, M. \& Sengur, A. Efficient deep features selections and classification for flower species recognition. Meas. J. Int. Meas. Confed. 137, 7-13 (2019).

62. Rodríguez, F. J., García, A., Pardo, P. J., Chávez, F. \& Luque-Baena, R. M. Study and classification of plum varieties using image analysis and deep learning techniques. Prog. Artif. Intell. 7, 119-127 (2018).

63. Pereira, C. S., Morais, R. \& Reis, M. J. C. S. Deep learning techniques for grape plant species identification in natural images. Sensors (Switzerland) 19, https:// doi.org/10.3390/s19224850 (2019).

64. Ponce, J. M., Aquino, A. \& Andújar, J. M. Olive-fruit variety classification by means of image processing and convolutional neural networks. IEEE Access $\mathbf{7}$, 147629 (2019).

65. Fernandes, A. M. et al. Grapevine variety identification using "Big Data" collected with miniaturized spectrometer combined with support vector machines and convolutional neural networks. Comput. Electron. Agric. 163, 104855 (2019).

66. Kang, H. \& Chen, C. Fruit detection and segmentation for apple harvesting using visual sensor in orchards. Sensors (Switzerland) 19, https://doi.org/ 10.3390/s19204599 (2019).

67. Kang, H. \& Chen, C. Fast implementation of real-time fruit detection in apple orchards using deep learning. Comput. Electron. Agric. 168, 105108 (2020).

68. Gené-Mola, J. et al. Fruit detection and 3D location using instance segmentation neural networks and structure-from-motion photogrammetry. Comput. Electron. Agric. 169, 105165 (2020).

69. Mao, S. et al. Automatic cucumber recognition algorithm for harvesting robots in the natural environment using deep learning and multi-feature fusion. Comput. Electron. Agric. 170, 105254 (2020).

70. Quiroz, I. A. \& Alférez, G. H. Image recognition of Legacy blueberries in a Chilean smart farm through deep learning. Comput. Electron. Agric. 168 105044 (2020).

71. Csillik, O., Cherbini, J., Johnson, R., Lyons, A. \& Kelly, M. Identification of citrus trees from unmanned aerial vehicle imagery using convolutional neural networks. Drones 2, 39 (2018).

72. Ampatzidis, Y. \& Partel, V. UAV-based high throughput phenotyping in citrus utilizing multispectral imaging and artificial intelligence. Remote Sens. 11 https://doi.org/10.3390/rs11040410 (2019). 
73. Sun, J. et al. Detection of key organs in tomato based on deep migration learning in a complex background. Agriculture 8, https://doi.org/10.3390/ agriculture8120196 (2018)

74. Shi, R., Li, T. \& Yamaguchi, Y. An attribution-based pruning method for realtime mango detection with YOLO network. Comput. Electron. Agric. 169, 105214 (2020).

75. Zhang, C., Li, H., Wang, X. \& Yang X. Cross-scene crowd counting via deep convolutional neural networks. Proc. IEEE Comput. Soc. Conf. Comput. Vis. Pattern Recognit. 833-841 (IEEE, 2015).

76. Lu, H., Cao, Z., Xiao, Y., Zhuang, B. \& Shen, C. TasselNet: counting maize tassels in the wild via local counts regression network. Plant Methods 13, 1-17 (2017).

77. Apolo-Apolo, O. E., Martínez-Guanter, J., Egea, G., Raja, P. \& Pérez-Ruiz, M. Deep learning techniques for estimation of the yield and size of citrus fruits using a UAV. Eur. J. Agron. 115, 126030 (2020).

78. Neupane, B., Horanont, T. \& Hung, N. D. Deep learning based banana plant detection and counting using high-resolution red-green-blue (RGB) images collected from unmanned aerial vehicle (UAV). PLOS ONE 14, 1-22 (2019).

79. Wang, Z., Walsh, K. \& Koirala, A. Mango fruit load estimation using a video based MangoYOLO_Kalman filter-hungarian algorithm method. Sensors (Switzerland) 19, https://doi.org/10.3390/s19122742 (2019).

80. Rahnemoonfar, M. \& Sheppard, C. Deep count: fruit counting based on deep simulated learning. Sensors (Switz.) 17, 1-12 (2017).

81. Afonso, M. et al. Tomato fruit detection and counting in greenhouses using deep learning. Front. Plant Sci. 11, 1-12 (2020).

82. Wang, Z., Hu, M. \& Zhai, G. Application of deep learning architectures for accurate and rapid detection of internal mechanical damage of blueberry using hyperspectral transmittance data. Sensors (Switz.) 18, 1-14 (2018).

83. Ucat, R. C. \& Cruz, J. C. D. Postharvest grading classification of cavendish banana using deep learning and tensorflow. 2019 Int Symp. Multimed. Commun. Technol. ISMAC 2019 2019. https://doi.org/ 10.1109/ISMAC.2019.8836129 (2019).

84. Bai, Y., Xiong, Y., Huang, J., Zhou, J. \& Zhang, B. Accurate prediction of soluble solid content of apples from multiple geographical regions by combining deep learning with spectral fingerprint features. Postharvest Biol. Technol. 156 110943 (2019).

85. Zhang, M., Jiang, Y., Li, C. \& Yang, F. Fully convolutional networks for blueberry bruising and calyx segmentation using hyperspectral transmittance imaging. Biosyst. Eng. 192, 159-175 (2020).

86. Liu, Z., He, Y., Cen, H. \& Lu, R. Deep feature representation with stacked sparse auto-encoder and convolutional neural network for hyperspectral imagingbased detection of cucumber defects. Trans. ASABE 61, 1-4 (2018).

87. Feng, L. et al. Detection of subtle bruises on winter jujube using hyperspectral imaging with pixel-wise deep learning method. IEEE Access 7, 64494-64505 (2019).

88. Lashgari, M., Imanmehr, A. \& Tavakoli, H. Fusion of acoustic sensing and deep learning techniques for apple mealiness detection. J. Food Sci. Technol. https://doi.org/10.1007/s13197-020-04259-y (2020).

89. Itakura, K., Saito, Y., Suzuki, T., Kondo, N. \& Hosoi, F. Estimation of citrus maturity with fluorescence spectroscopy using deep learning. Horticulturae $\mathbf{5}$, 1-9 (2019).

90. Ghosal, S. et al. An explainable deep machine vision framework for plant stress phenotyping. Proc. Natl Acad. Sci. USA 115, 4613-4618 (2018).

91. Fuentes, A., Yoon, S., Kim, S. C. \& Park, D. S. A robust deep-learning-based detector for real-time tomato plant diseases and pests recognition. Sensors (Switzerland) 17, https://doi.org/10.3390/s17092022 (2017).

92. Karthik, R. et al. Attention embedded residual CNN for disease detection in tomato leaves. Appl. Soft. Comput. J. 86, 105933 (2020).

93. Ma, J. et al. A recognition method for cucumber diseases using leaf symptom images based on deep convolutional neural network. Comput. Electron. Agric. 154, 18-24 (2018).
94. Aravind, K. R., Raja, P., Ashiwin, R. \& Mukesh, K. V. Disease classification in solanum melongena using deep learning. Spanish J. Agric. Res. 17, https://doi. org/10.5424/sjar/2019173-14762 (2019).

95. Zhang, S., Zhang, S., Zhang, C., Wang, X. \& Shi, Y. Cucumber leaf disease identification with global pooling dilated convolutional neural network. Comput. Electron. Agric. 162, 422-430 (2019).

96. Kim, W. S., Lee, D. H. \& Kim, Y. J. Machine vision-based automatic disease symptom detection of onion downy mildew. Comput. Electron. Agric. 168, 105099 (2020)

97. Oppenheim, D., Shani, G., Erlich, O. \& Tsror, L. Using deep learning for imagebased potato tuber disease detection. Phytopathology 109, 1083-1087 (2019).

98. Alruwaili, M., Alanazi, S., El-Ghany, S. A. \& Shehab, A. An efficient deep learning model for olive diseases detection. Int. J. Adv. Comput. Sci. Appl. 10 486-492 (2019).

99. Zhong, Y. \& Zhao, M. Research on deep learning in apple leaf disease recognition. Comput. Electron. Agric. 168, 105146 (2020).

100. Sun, Y., Wei, K., Liu, Q., Pan, L. \& Tu, K. Classification and discrimination of different fungal diseases of three infection levels on peaches using hyperspectral reflectance imaging analysis. Sensors (Switzerland) 18, https://doi.org/ 10.3390/s18041295 (2018)

101. Tian, Y., Yang, G., Wang, Z., Li, E. \& Liang, Z. Instance segmentation of apple flowers using the improved mask R-CNN model. Biosyst. Eng. 193, 264-278 (2020).

102. Tian, Y. et al. Apple detection during different growth stages in orchards using the improved YOLO-V3 model. Comput. Electron. Agric. 157, 417-426 (2019).

103. Wang, Z., Underwood, J. \& Walsh, K. B. Machine vision assessment of mango orchard flowering. Comput. Electron. Agric. 151, 501-511 (2018).

104. Koirala, A., Walsh, K. B., Wang, Z. \& Anderson N. Deep learning for mango (Mangifera indica) panicle stage classification. Agronomy 10, https://doi.org/ 10.3390/agronomy10010143. (2020).

105. Bauer, A. et al. Combining computer vision and deep learning to enable ultra-scale aerial phenotyping and precision agriculture: a case study of lettuce production. Hortic. Res. 6, https://doi.org/10.1038/s41438-019-0151-5. (2019).

106. Montesinos-López, O. A. et al. Multi-Trait, multi-environment genomic prediction of durum wheat with genomic best linear unbiased predictor and deep learning methods. Front. Plant Sci. 10, https://doi.org/10.3389/ fpls.2019.01311 (2019).

107. Tsaftaris, S. A., Minervini, M. \& Scharr, H. Machine learning for plant phenotyping needs image processing. Trends Plant Sci. 21, 989-991 (2016).

108. Gebbers, R. \& Adamchuk, V. I. Precision agriculture and food security. Science 327, 828-831 (2010).

109. Majeed, Y. et al. Apple tree trunk and branch segmentation for automatic trellis training using convolutional neural network based semantic segmentation. IFAC-PapersOnLine 51, 75-80 (2018).

110. LeCun, Y., Bottou, L., Bengio, Y. \& Haffner, P. Gradient-based learning applied to document recognition. Proc. IEEE 86, 2278-2323 (1998).

111. Krizhevsky, A., Sutskever, I., \& Hinton, G. E. In: Advances in Neural Information Processing Systems. 1097-1105 (MIT Press, 2012).

112. Simonyan, K. \& Zisserman, A. Very deep convolutional networks for large-scale image recognition. In: 3rd International Conference on Learning Representations, ICLR 2015-Conference Track Proceedings. pp 1-14 (San Diego, 2015)

113. Szegedy, C. et al. Going deeper with convolutions. In: Proceedings of the IEEE Computer Society Conference on Computer Vision and Pattern Recognition. pp 1-9 (Boston, 2015)

114. He, K., Zhang, X., Ren, S. \& Sun, J. Deep residual learning for image recognition. In: Proceedings of the IEEE Computer Society Conference on Computer Vision and Pattern Recognition. pp 770-778 (Las Vegas, 2016). 